\title{
MAXIMAL SUBGROUPS OF MULTI-EDGE SPINAL GROUPS
}

\author{
THEOFANIS ALEXOUDAS, BENJAMIN KLOPSCH, \\ AND ANITHA THILLAISUNDARAM
}

\begin{abstract}
A multi-edge spinal group is a subgroup of the automorphism group of a regular $p$-adic rooted tree, generated by one rooted automorphism and a finite number of directed automorphisms sharing a common directing path. We prove that torsion multi-edge spinal groups do not have maximal subgroups of infinite index. This generalizes a result of Pervova for GGS-groups.
\end{abstract}

\section{INTRODUCTION}

Branch groups are groups acting spherically transitively on a spherically homogeneous infinite rooted tree and having subnormal subgroup structure similar to the corresponding structure in the full group of automorphisms of the tree. Early constructions were produced by Grigorchuk [5] and Gupta and Sidki [8], and they were generalized to so-called GGS-groups. The class of branch groups provides important and easily describable examples for finitely generated groups of intermediate word growth, or for finitely generated infinite torsion groups; cf. the General Burnside Problem.

We deal here with multi-edge spinal groups acting on the regular $p$ adic rooted tree $T$, for $p$ an odd prime. A multi-edge spinal group $G=$ $\left\langle a, b_{1}, \ldots, b_{r}\right\rangle$ is generated by a rooted automorphism $a$ and a finite number of directed automorphisms $b_{1}, \ldots, b_{r}$, for some $r \in\{1, \ldots, p-1\}$; see Section 3 for details. These groups generalize GGS-groups, which correspond to the special case $r=1$. In particular, every torsion multi-edge spinal group is an infinite $p$-group. Moreover we show that, apart from one possible exception, multi-edge spinal groups are branch; see Proposition 3.7.

Pervova [11, 12] proved that the Grigorchuk group and torsion GGSgroups do not contain maximal subgroups of infinite index. Equivalently, these groups do not contain proper dense subgroups with respect to the profinite topology. On the other hand - prompted by a question of Grigorchuk, Bartholdi and Šuniḱk [2] - Bondarenko gave in [3] a non-constructive example of a finitely generated branch group that does have maximal subgroups of infinite index. Hence we face the following problem.

2010 Mathematics Subject Classification. Primary 20E08; Secondary 20E28.

Key words and phrases. multi-edge spinal groups, branch groups, maximal subgroups. 
Problem 1.1. Characterize among finitely generated branch groups those that possess maximal subgroups of infinite index and those that do not.

In particular, Bondarenko's method - by itself - does not apply to groups acting on the regular $p$-adic rooted tree $T$ that are residually finite- $p$. It is natural to test how far Pervova's results in [12] can be extended and multi-edge spinal groups form a suitable generalization of GGS-groups.

Theorem 1.2. Let $G$ be a multi-edge spinal group acting on the regular $p$-adic rooted tree, for $p$ an odd prime, and suppose that $G$ is torsion. Then every maximal subgroup of $G$ is normal of finite index $p$.

As indicated in [12, one motivation for our investigation comes from a conjecture of Passman concerning the group algebra $K[G]$ of a finitely generated group $G$ over a field $K$ with char $K=p$. The conjecture states that, if the Jacobson radical $\mathcal{J}(K[G])$ coincides with the augmentation ideal $\mathcal{A}(K[G])$ then $G$ is a finite $p$-group; see [9, Conjecture 6.1]. In [9], Passman showed that if $\mathcal{J}(K[G])=\mathcal{A}(K[G])$ then $G$ is a $p$-group and every maximal subgroup of $G$ is normal of index $p$. Hence multi-edge spinal groups that are torsion yield natural candidates for testing Passman's conjecture. It is important to widen this class of candidates, as even the Gupta-Sidki group for $p=3$ does not satisfy $\mathcal{J}(K[G])=\mathcal{A}(K[G])$; this follows from [14.

Grigorchuk and Wilson [7] have generalized Pervova's results in [11, 12. by means of commensurability. Two groups are said to be commensurable if they have isomorphic subgroups of finite index. Let $G$ be as in Theorem 1.2 . From [7, Lemma 1], it follows that whenever $G$ is a subgroup of finite index in a group $H$ then $H$ does not have maximal subgroups of infinite index. Using this and [7, Lemma 3] we derive the following consequence of Theorem 1.2 .

Corollary 1.3. Let $G$ be as in Theorem 1.2. If $H$ is a group commensurable with $G$ then every maximal subgroup of $H$ has finite index in $H$.

Finally, we remark that most parts of the proof of Theorem 1.2 go through under the assumption that the group is just infinite and not necessarily torsion. One may therefore speculate that, in fact, every just infinite multiedge spinal group has the property that all its maximal subgroups are of finite index.

\section{Preliminaries}

In the present section we recall the notion of branch groups and establish prerequisites for the rest of the paper. For more details, see [2, 6].

2.1. The regular $p$-adic rooted tree and its automorphisms. Let $T$ the regular $p$-adic rooted tree, for an odd prime $p$. Let $X$ be an alphabet on $p$ letters, e.g., $X=\{1,2, \ldots, p\}$. The set of vertices of $T$ can be identified with the free monoid $\bar{X}$; in particular, the root of $T$ corresponds to the empty word $\varnothing$. For each word $v \in \bar{X}$ and letter $x$, an edge connects $v$ to $v x$. There is a natural length function on $\bar{X}$, and the words $w$ of length 
$|w|=n$, representing vertices that are at distance $n$ from the root, constitute the nth layer of the tree. The tree is called regular because all vertices have the same out-degree $p$, and the boundary $\partial T$ consisting of all infinite rooted paths is in one-to-one correspondence with the $p$-adic integers. More generally, one considers rooted trees that are not necessarily regular, but spherically homogeneous, meaning that vertices of the same length have the same degree.

We write $T_{u}$ for the full rooted subtree of $T$ that has its root at a vertex $u$ and includes all vertices $v$ with $u$ a prefix of $v$. As $T$ is regular $p$-adic, for any two vertices $u$ and $v$ the subtrees $T_{u}$ and $T_{v}$ are isomorphic under the map that deletes the prefix $u$ and replaces it by the prefix $v$. We refer to this identification as the natural identification of subtrees and write $T_{n}$ to denote the subtree rooted at a generic vertex of level $n$.

We observe that every automorphism of $T$ fixes the root and that the orbits of $\operatorname{Aut}(T)$ on the vertices of the tree $T$ are precisely its layers. Consider an automorphism $f \in \operatorname{Aut}(T)$. The image of a vertex $u$ under $f$ is denoted by $u^{f}$. For a vertex $u$, thought of as a word over $X$, and a letter $x \in X$ we have $(u x)^{f}=u^{f} x^{\prime}$ where $x^{\prime} \in X$ is uniquely determined by $u$ and $f$. This induces a permutation $f(u)$ of $X$ so that

$$
(u x)^{f}=u^{f} x^{f(u)} .
$$

The automorphism $f$ is called rooted if $f(u)=1$ for $u \neq \varnothing$. It is called directed, with directing path $\ell \in \partial T$, if the support $\{u \mid f(u) \neq 1\}$ of its labelling is infinite and contains only vertices at distance 1 from $\ell$.

The section of $f$ at a vertex $u$ is the unique automorphism $f_{u}$ of $T \cong T_{|u|}$ given by the condition $(u v)^{f}=u^{f} v^{f_{u}}$ for $v \in \bar{X}$.

2.2. Subgroups of $\operatorname{Aut}(T)$. Let $G$ be a subgroup of $\operatorname{Aut}(T)$ acting spherically transitively, i.e., transitively on every layer of $T$. The vertex stabilizer $\operatorname{Stab}_{G}(u)$ is the subgroup consisting of elements in $G$ that fix the vertex $u$. For $n \in \mathbb{N}$, the $n$th level stabilizer $\operatorname{Stab}_{G}(n)=\cap_{|v|=n} \operatorname{Stab}_{G}(v)$ is the subgroup consisting of automorphisms that fix all vertices at level $n$. Note that elements in $\operatorname{Stab}_{G}(n)$ fix all vertices up to level $n$ and that $\operatorname{Stab}_{G}(n)$ has finite index in $G$.

The full automorphism group $\operatorname{Aut}(T)$ is a profinite group. Indeed,

$$
\operatorname{Aut}(T)=\lim _{n \rightarrow \infty} \operatorname{Aut}\left(T_{[n]}\right)
$$

where $T_{[n]}$ denotes the subtree of $T$ on the finitely many vertices up to level $n$. The topology of $\operatorname{Aut}(T)$ is defined by the open subgroups $\operatorname{Stab}_{\operatorname{Aut}(T)}(n)$, $n \in \mathbb{N}$. The level stabilizers $\operatorname{Stab}_{G}(n), n \in \mathbb{N}$, form a natural family of principal congruence subgroups for $G$. The subgroup $G$ of $\operatorname{Aut}(T)$ has the congruence subgroup property if the profinite topology and the congruence topology on $G$ coincide, i.e., if for every subgroup $H$ of finite index in $G$, there exists some $n$ such that $\operatorname{Stab}_{G}(n) \subseteq H$. 
Every $g \in \operatorname{Stab}_{\operatorname{Aut}(T)}(n)$ can be identified with a collection $g_{1}, \ldots, g_{p^{n}}$ of elements of $\operatorname{Aut}\left(T_{n}\right)$, where $p^{n}$ is the number of vertices at level $n$. Indeed, denoting by $u_{1}, \ldots, u_{p^{n}}$ the vertices of $T$ at level $n$, there is a natural isomorphism

$$
\operatorname{Stab}_{\text {Aut }(T)}(n) \cong \prod_{i=1}^{p^{n}} \operatorname{Aut}\left(T_{u_{i}}\right) \cong \operatorname{Aut}\left(T_{n}\right) \times p^{n} . \times \operatorname{Aut}\left(T_{n}\right) .
$$

Since $T$ is regular, $\operatorname{Aut}\left(T_{n}\right)$ is isomorphic to $\operatorname{Aut}(T)$ after the natural identification of subtrees. Therefore the decomposition $g=\left(g_{1}, \ldots, g_{p^{n}}\right)$ defines an embedding

$$
\psi_{n}: \operatorname{Stab}_{\operatorname{Aut}(T)}(n) \rightarrow \prod_{i=1}^{p^{n}} \operatorname{Aut}\left(T_{u_{i}}\right) \cong \operatorname{Aut}(T) \times \stackrel{p}{n}^{n} \times \operatorname{Aut}(T) .
$$

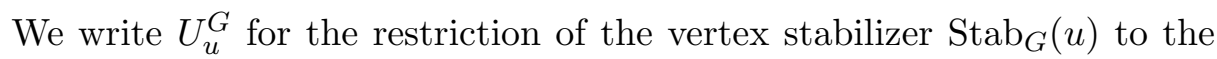
subtree rooted at a vertex $u$. Since $G$ acts spherically transitively, the vertex stabilizers at every level are conjugate under $G$. We write $U_{n}^{G}$ for the common isomorphism type of the restriction of the $n$th level vertex stabilizers, and we call it the $n$th upper companion group of $G$. We say that $G$ is fractal if every upper companion group $U_{n}^{G}$ coincides with the group $G$, after the natural identification of subtrees.

Next, the subgroup $\operatorname{Rstab}_{G}(u)$, consisting of all automorphisms in $G$ that fix all vertices $v$ of $T$ not having $u$ as a prefix, is called the rigid vertex stabilizer of $u$ in $G$. For a vertex $u \in T$, we write $\operatorname{Rstab}_{G}(u)_{u}$ for the restriction of the rigid vertex stabilizer to the subtree rooted at $u$. The rigid nth level stabilizer is the product

$$
\operatorname{Rstab}_{G}(n)=\prod_{i=1}^{p^{n}} \operatorname{Rstab}_{G}\left(u_{i}\right) \unlhd G
$$

of the rigid vertex stabilizers of the vertices $u_{1}, \ldots, u_{p^{n}}$ at level $n$. Since $G$ acts spherically transitively, the rigid vertex stabilizers at each level are conjugate under $G$. The common isomorphism type $L_{n}^{G}$ of the $n$th level rigid vertex stabilizers is called the $n$th lower companion group of $G$.

2.3. Branch groups. More generally, we recall that a spherically homogeneous infinite rooted tree $T=T_{\bar{m}}$ is constructed over a sequence of alphabets $X_{1}, X_{2}, \ldots$ with $\left|X_{i}\right|=m_{i} \geq 2$, where $\bar{m}=\left(m_{n}\right)_{n=1}^{\infty}$ is a sequence of natural numbers, in such a way that all vertices at the same level $n-1$ have the same out-degree $m_{n}$. In the case of a regular $p$-adic rooted tree, for $p$ an odd prime, the branching sequence is constant: $\bar{m}=(p, p, \ldots)$.

Definition 2.1 ([6]). A group $G$ is a branch group, if there is a spherically homogeneous rooted tree $T=T_{\bar{m}}$, with branching sequence $\bar{m}=\left(m_{n}\right)_{n=1}^{\infty}$, and an embedding $G \hookrightarrow \operatorname{Aut}(T)$ such that

(1) the group $G$ acts transitively on each layer of the tree;

(2) for each level $n$ there exists a subgroup $L_{n}$ of the automorphism group $\operatorname{Aut}\left(T_{n}\right)$ of the full subtree $T_{n}$ rooted at a level $n$ vertex such that the direct product

$$
H_{n}=L_{n}^{(1)} \times \ldots \times L_{n}^{\left(N_{n}\right)} \leq \operatorname{Stab}_{\operatorname{Aut}(T)}(n), \text { where } L_{n}^{(j)} \cong L_{n},
$$


of $N_{n}=\prod_{i=1}^{n} m_{i}$ copies of $L_{n}$ is normal and of finite index in $G$.

There exists an alternative and more intrinsic algebraic definition, which can also be found in [6]. For a fixed embedding $G \hookrightarrow \operatorname{Aut}(T)$, the pair $\left(\left(L_{n}\right)_{n=1}^{\infty},\left(H_{n}\right)_{n=1}^{\infty}\right)$ is called a branch structure. If $G$ is branch then a 'standard' branch structure is given by $\left(\left(L_{n}^{G}\right)_{n=1}^{\infty},\left(\operatorname{Rstab}_{G}(n)\right)_{n=1}^{\infty}\right)$. Thus condi-

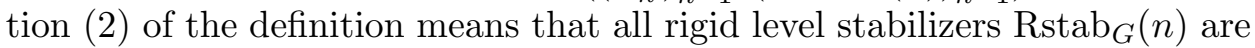
of finite index in $G$.

\section{Multi-EDGe SPINAL GROUPS}

Let $T$ be the regular $p$-adic rooted tree, for an odd prime $p$. The vertices of $T$ can be identified with words over an alphabet $X$ of size $p$; sometimes it is convenient to label them explicitly by finite sequences in $\{1, \ldots, p\}$. Let $\ell=\left(l_{n}\right)_{n=0}^{\infty}$ be an infinite path in $T$ starting at the root, with $l_{n}=x_{1} \cdots x_{n}$ where $x_{1}, \ldots, x_{n} \in X$. For every $n \geq 1$ and $y \in X \backslash\left\{x_{n}\right\}$, we denote by $s_{n, y}$ the immediate descendants of $l_{n-1}$ not lying in $\ell$. The doubly indexed family $S=\left(s_{n, y}\right)_{n, y}$ is a multi-edge spine in $T$. Recall that $\operatorname{Aut}(T)$ acts transitively within the layers of $T$, and in particular, on the boundary $\partial T$. Hence, conjugating by an element of $\operatorname{Aut}(T)$, we may choose, for simplicity, the spine to be associated to the rightmost infinite path $\left(\varnothing, u_{p}, u_{p p}, \ldots\right)$ starting at the root vertex of the tree.

3.1. Construction of multi-edge spinal groups. By $a$ we denote the rooted automorphism, corresponding to the $p$-cycle $(12 \ldots p) \in \operatorname{Sym}(p)$, that cyclically permutes the vertices $u_{1}, \ldots, u_{p}$ of the first level. Recall the coordinate map

$\psi_{1}: \operatorname{Stab}_{\text {Aut }(T)}(1) \rightarrow \operatorname{Aut}\left(T_{u_{1}}\right) \times \ldots \times \operatorname{Aut}\left(T_{u_{p}}\right) \cong \operatorname{Aut}(T) \times \stackrel{p}{ } . \times \operatorname{Aut}(T)$.

Given $r \in \mathbb{N}$ and a finite $r$-tuple $\mathbf{E}$ of $(\mathbb{Z} / p \mathbb{Z})$-linearly independent vectors

$$
\mathbf{e}_{i}=\left(e_{i, 1}, e_{i, 2}, \ldots, e_{i, p-1}\right) \in(\mathbb{Z} / p \mathbb{Z})^{p-1}, \quad i \in\{1, \ldots, r\},
$$

we recursively define directed automorphisms $b_{1}, \ldots, b_{r} \in \operatorname{Stab}_{\operatorname{Aut}(T)}(1)$ via

$$
\psi_{1}\left(b_{i}\right)=\left(a^{e_{i, 1}}, a^{e_{i, 2}}, \ldots, a^{e_{i, p-1}}, b_{i}\right), \quad i \in\{1, \ldots, r\} .
$$

We call the subgroup $G=G_{\mathbf{E}}=\left\langle a, b_{1}, \ldots, b_{r}\right\rangle$ of $\operatorname{Aut}(T)$ the multi-edge spinal group associated to the defining vectors $\mathbf{E}$. We observe that $\langle a\rangle \cong C_{p}$ and $\left\langle b_{1}, \ldots, b_{r}\right\rangle \cong C_{p}^{r}$ are elementary abelian $p$-groups.

By choosing only one vector

$$
\mathbf{e}=\left(e_{1}, \ldots, e_{p-1}\right) \in(\mathbb{Z} / p \mathbb{Z})^{p-1}
$$

and defining an automorphism $b$ of $\operatorname{Aut}(T)$ via

$$
\psi_{1}(b)=\left(a^{e_{1}}, \ldots, a^{e_{p-1}}, b\right)
$$

we obtain the GGS-group $G_{(\mathbf{e})}=\langle a, b\rangle$ corresponding to the defining vector e. For instance, the Gupta-Sidki group for the prime $p$ arises by choosing $\mathbf{e}=(1,-1,0, \ldots, 0) ;$ see $[8]$. 
3.2. General properties of multi-edge spinal groups. The proof of the following result is straightforward; details may be found in [1].

Proposition 3.1. Let $G$ be a multi-edge spinal group. Then every section of every element of $G$ is contained in $G$. Moreover, $G$ acts spherically transitively on the tree $T$ and $G$ is fractal.

The next theorem, adapted to the present context, gives necessary and sufficient conditions for a multi-edge spinal group to be periodic.

Theorem $3.2([6,15])$. Let $G_{\mathbf{E}}=\left\langle a, b_{1}, \ldots, b_{r}\right\rangle$ be a multi-edge spinal group corresponding to an $r$-tuple of defining vectors $\mathbf{E}=\left(\mathbf{e}_{i}\right)_{i=1}^{r}$. Then $G$ is an infinite p-group if and only if for every $\mathbf{e}_{i}=\left(e_{i, 1}, \ldots, e_{i, p-1}\right)$,

$$
\sum_{j=1}^{p-1} e_{i, j} \equiv 0 \quad(\bmod p)
$$

The next lemma shows: by a 'change of coordinates', we can arrange that $e_{1,1}=1$ in the defining vector $\mathbf{e}_{1}$ of a multi-edge spinal group $G_{\mathbf{E}}$.

Lemma 3.3. Let $G=G_{\mathbf{E}}$ be a multi-edge spinal group. Then there exists an automorphism $f \in \operatorname{Aut}(T)$ of the form $f=f_{0} f_{1}=f_{1} f_{0}$, where $f_{0}$ is a rooted automorphism corresponding to a permutation $\pi \in \operatorname{Sym}(p)$ and $f_{1} \in$ $\operatorname{Stab}_{G}(1)$ with $\psi_{1}\left(f_{1}\right)=(f, \ldots, f)$, such that $G^{f}=G_{\widetilde{\mathbf{E}}}=\left\langle a, \tilde{b}_{1}, \ldots, \tilde{b}_{r}\right\rangle$ is a multi-edge spinal group generated by the rooted automorphism a and directed automorphisms $\tilde{b}_{1}=b_{1}^{f}, \ldots, \tilde{b}_{r}=b_{r}^{f}$ satisfying $\psi_{1}\left(\tilde{b}_{i}\right)=\left(a^{\tilde{e}_{i, 1}}, \ldots, a^{\tilde{e}_{i, p-1}}, \tilde{b}_{i}\right)$ for $i \in\{1, \ldots, r\}$ with $\tilde{e}_{1,1}=1$.

Proof. Since the defining vectors $\mathbf{e}_{1}, \ldots, \mathbf{e}_{r}$ for $G$ are linearly independent over $\mathbb{Z} / p \mathbb{Z}$, each $\mathbf{e}_{i}$ satisfies

$$
\mathbf{e}_{i}=\left(e_{i, 1}, \ldots, e_{i, p-1}\right) \not \equiv \mathbf{0} \quad(\bmod p) .
$$

In particular $\mathbf{e}_{1} \not \equiv \mathbf{0}(\bmod p)$. Without loss of generality, assume that $e_{1, k} \equiv$ $k$ for some $k \in\{1, \ldots, p-1\}$; otherwise we replace $b_{1}$ by a power of itself. Then there exists some $l \in\{1, \ldots, p-1\}$ such that $k l \equiv 1(\bmod p)$. Define a permutation $\pi \in \operatorname{Sym}(p)$ by $x \pi=l x$, where $x \in\{1, \ldots, p\}$ represents a vertex in the first level of the tree $T$. Observe that $x \pi^{-1}=k x$ for all $x \in\{1, \ldots, p\}$. We consider the automorphism $f=f_{0} f_{1}=f_{1} f_{0} \in \operatorname{Aut}(T)$, where $f_{0}$ is a rooted automorphism corresponding to the permutation $\pi \in$ $\operatorname{Sym}(p)$ and $f_{1} \in \operatorname{Stab}_{G}(1)$ is given by $\psi_{1}\left(f_{1}\right)=(f, \ldots, f)$.

Set $\tilde{a}=\left(a^{k}\right)^{f}=\left(a^{k}\right)^{f_{0}}$. Then, for all $x \in\{1, \ldots, p\}$,

$$
x^{\tilde{a}} \equiv x^{f_{0}^{-1} a^{k} f_{0}} \equiv(k x)^{a^{k} f_{0}} \equiv(k x+k)^{f_{0}} \equiv(k x+k) l \equiv x+1 \equiv x^{a} \quad(\bmod p) .
$$

Hence $\tilde{a}=a$. It follows that $a=\left(a^{k}\right)^{f}=\left(a^{f}\right)^{k}$, implying $a^{f}=a^{l}$.

Setting $\tilde{b}_{i}=\left(b_{i}\right)^{f}$ for $i \in\{1, \ldots, r\}$, we obtain

$$
\begin{aligned}
\tilde{b}_{i} & =\left(f^{-1}, \ldots, f^{-1}\right)(a^{e_{i, k}}, \ldots, \underbrace{a^{e_{i, 1}}}_{l^{\text {th }} \text { resp. }(l+1)^{\text {th }} \text { coord. }}, \underbrace{a^{e_{i, k+1}}}, \ldots, a^{e_{i, p-k}}, b_{i})(f, \ldots, f) \\
& =\left(a^{l e_{i, k}}, \ldots, a^{l e_{i, p-k}}, \tilde{b}_{i}\right) .
\end{aligned}
$$


In particular, $\tilde{e}_{1,1} \equiv l e_{1, k} \equiv l k \equiv 1(\bmod p)$. Thus $f$ as defined above has the required properties.

In preparation for Proposition 3.5 we establish the following lemma.

Lemma 3.4. Let $G_{\mathbf{E}}=\left\langle a, b_{1}, \ldots, b_{r}\right\rangle$ be a multi-edge spinal group associated to an $r$-tuple $\mathbf{E}$ with $r \geq 2$. Then there exists an $r$-tuple of defining vectors $\widetilde{\mathbf{E}}$ such that $G_{\widetilde{\mathbf{E}}}$ is conjugate to $G_{\mathbf{E}}$ by an element $f \in \operatorname{Aut}(T)$ as in Lemma 3.3 and the following hold:

(1) $\tilde{e}_{i, 1} \equiv 1(\bmod p)$ for each $i \in\{1, \ldots, r\}$;

(2) if $r=2$ and $p=3$, then $\tilde{\mathbf{e}}_{1}=(1,0), \tilde{\mathbf{e}}_{2}=(1,1)$;

(3) if $r=2$ and $p>3$, then either

(a) for each $i \in\{1,2\}$ there exists $k \in\{2, \ldots, p-2\}$ such that $\tilde{e}_{i, k-1} \tilde{e}_{i, k+1} \not \equiv \tilde{e}_{i, k}^{2}(\bmod p)$, or

(b) $\tilde{\mathbf{e}}_{1}=(1,0, \ldots, 0,0), \tilde{\mathbf{e}}_{2}=(1,0, \ldots, 0,1)$;

(4) if $r \geq 3$ then for each $i \in\{1, \ldots, r\}$ there exists $k \in\{2, \ldots, p-2\}$ such that $\tilde{e}_{i, k-1} \tilde{e}_{i, k+1} \not \equiv \tilde{e}_{i, k}^{2}(\bmod p)$.

Proof. We split the proof into two cases: $r \geq 3$ and $r=2$.

Case 1: $r \geq 3$. Observe that $p \geq 5$ and consider the $r \times(p-1)$-matrix

$$
M(\mathbf{E})=\left(\begin{array}{ccc}
e_{1,1} & \ldots & e_{1, p-1} \\
e_{2,1} & \ldots & e_{2, p-1} \\
\vdots & \ddots & \vdots \\
e_{r, 1} & \ldots & e_{r, p-1}
\end{array}\right)
$$

encoding the defining vectors for the group $G_{\mathbf{E}}$. By Lemma 3.3. we may assume that $e_{1,1} \not \equiv 0$. Using elementary row operations, we transform $M(\mathbf{E})$ into reduced row-echelon form:

$$
\left(\begin{array}{cccccccccccc}
1 & a_{1} & \ldots & a_{m} & 0 & * & \ldots & * & 0 & * & \ldots & * \\
0 & 0 & \ldots & 0 & 1 & * & \ldots & * & 0 & * & \ldots & * \\
\vdots & \vdots & & \vdots & & \vdots & & \vdots & & \vdots & & \vdots \\
0 & 0 & \ldots & 0 & 0 & 0 & \ldots & 0 & 1 & * & \ldots & *
\end{array}\right)
$$

where $m \geq 0, a_{1}, \ldots, a_{m} \in \mathbb{Z} / p \mathbb{Z}$ and the symbols $*$ denote other, unspecified elements of $\mathbb{Z} / p \mathbb{Z}$. Adding the 1 st row to all other rows, we obtain

$$
M(\widetilde{\mathbf{E}})=\left(\begin{array}{cccccccccccc}
1 & a_{1} & \ldots & a_{m} & 0 & * & \ldots & * & 0 & * & \ldots & * \\
1 & a_{1} & \ldots & a_{m} & 1 & * & \ldots & * & 0 & * & \ldots & * \\
\vdots & \vdots & & \vdots & & \vdots & & \vdots & & \vdots & & \vdots \\
1 & a_{1} & \ldots & a_{m} & 0 & * & \ldots & * & 1 & * & \ldots & *
\end{array}\right)
$$

The row operations that we carried out yield a new set of generators for $\left\langle b_{1}, \ldots, b_{r}\right\rangle$, corresponding to an $r$-tuple $\widetilde{\mathbf{E}}$ of defining vectors that are encoded in the rows of $M(\widetilde{\mathbf{E}})$. 
Let $i \in\{1, \ldots, r\}$ and consider the $i$ th row of $M(\widetilde{\mathbf{E}})$. We identify two patterns which guarantee that the $i$ th row satisfies the condition in (4):

$$
\begin{aligned}
& \text { (A) } \quad(* \ldots * x y 0 * \ldots *), \\
& (\mathrm{B}) \quad(* \ldots * 0 y x * \ldots *),
\end{aligned}
$$

where $x, y \in \mathbb{Z} / p \mathbb{Z}$ with $y \not \equiv 0$ and the symbols $*$ again denote unspecified elements. Observe that, if the patterns (A) and (B) do not appear in the $i$ th row, then the row does not have any zero entries at all or must be of the form $(* 0 \ldots 0 *)$.

Suppose first that $2 \leq i \leq r-1$. In this case the $i$ th row contains at least one zero entry and cannot be of the form $(* 0 \ldots 0 *)$. Hence the pattern (A) or (B) occurs.

Next suppose that $i=r$ and assume that patterns (A) or (B) do not appear. As $r \geq 3$ the $r$ th row contains at least one zero entry and consequently has the form $\left(\begin{array}{llll}1 & 0 & \ldots & 0\end{array}\right)$. Changing generators, we may replace the $r$ th row by the $r$ th row minus the 2 nd row plus the 1st row, yielding

$$
\left(\begin{array}{ccccccccc}
1 & 0 & \ldots & 0 & -1 & * & \ldots & * & 1
\end{array}\right)
$$

with $m$ zeros between the entries 1 and -1 . If $m>0$ then pattern (B) occurs in this new row. Suppose that $m=0$. Then the row takes the form

$$
\left(\begin{array}{llllll}
1 & -1 & * & \ldots & * & 1
\end{array}\right) \text {. }
$$

For the condition in (4) to fail, we would need the row to be equal to $\left(\begin{array}{lllll}1-1 & 1-1 \ldots 1-1\end{array}\right)$ with the final entry being -1 as $p-1$ is even. This contradicts 3.3 .

Finally, suppose that $i=1$. Similarly as above, we assume that patterns (A) and (B) do not occur. Since it contains at least one zero entry, the 1st row is of the form

$$
\left(\begin{array}{lllll}
1 & 0 & \ldots & 0 & *
\end{array}\right)
$$

and we change generators as follows. Generically, we replace the 1st row by the 1st row plus the 2nd row minus the 3rd row. Only if $r=3$ and we already changed the $r$ th row as described above, we replace the 1st row by 2 times the 1st row minus the 3rd row. In any case, this gives a new 1st row:

$$
\left(\begin{array}{llllllllllll}
1 & 0 & \ldots & 0 & 1 & * & \ldots & * & -1 & * & \ldots & *
\end{array}\right)
$$

with $m$ zeros between the entries 1 and 1 . If $m>0$ then pattern (B) occurs. Suppose that $m=0$ so that the new row takes the form

$$
\left(\begin{array}{ccccccccc}
1 & 1 & * & \ldots & * & -1 & * & \ldots & *
\end{array}\right) \text {. }
$$

For the condition in (4) to fail, the row would have to be of the form $\left(\begin{array}{llll}1 & 1 & \ldots & 1\end{array}\right)$ contradicting (3.5).

Case 2: $r=2$. The statement in (2) for $p=3$ can clearly be achieved by a simple change of generators. Now we suppose that $p>3$. By Lemma 3.3, we may assume that $e_{1,1} \not \equiv 0$. Using elementary row operations, we transform 
the $2 \times(p-1)$-matrix $M(\mathbf{E})$ encoding the defining vectors into reduced row-echelon form

$$
\left(\begin{array}{llll}
1 & \mathbf{a} & 0 & \mathbf{b} \\
0 & \mathbf{0} & 1 & \mathbf{c}
\end{array}\right)
$$

where at most one of $\left(\begin{array}{l}\mathbf{a} \\ \mathbf{0}\end{array}\right)$ or $\left(\begin{array}{l}\mathbf{b} \\ \mathbf{c}\end{array}\right)$ could be the empty matrix. Further row operations, corresponding to multiplication on the left by $\left(\begin{array}{cc}1 & y \\ 1 & z\end{array}\right)$, where $y, z \in \mathbb{Z} / p \mathbb{Z}$ with $y \not \equiv z$ are to be specified below, yield

$$
M(\widetilde{\mathbf{E}})=\left(\begin{array}{cccc}
1 & \mathbf{a} & y & \mathbf{b}+y \mathbf{c} \\
1 & \mathbf{a} & z & \mathbf{b}+z \mathbf{c}
\end{array}\right)
$$

encoding an $r$-tuple $\widetilde{\mathbf{E}}$ of defining vectors for a new set of generators.

First suppose that $\mathbf{a}=\mathbf{0}$ is not empty and zero. If $\mathbf{b}=()$ then

$$
M(\widetilde{\mathbf{E}})=\left(\begin{array}{ccccc}
1 & 0 & \ldots & 0 & y \\
1 & 0 & \ldots & 0 & z
\end{array}\right)
$$

leads to $(3)(\mathbf{b})$. Otherwise, if $\mathbf{b} \neq()$, we choose $y \equiv 1$ and $z \equiv-1(\bmod p)$, yielding pattern (B) in both rows so that the condition in (3)(a) holds.

Next suppose that $\mathbf{a}=\left(a_{1} \ldots a_{m}\right) \neq \mathbf{0}$ is not empty and non-zero. Suppose further that the truncated rows $(1 \mathbf{a} y),(1 \mathbf{a} z)$ do not yet satisfy the condition in (3)(a). Then pattern (B) does not occur in these and a cannot have any zero entries. Consequently, there exists $\lambda \in \mathbb{Z} / p \mathbb{Z} \backslash\{0\}$ such that $M(\widetilde{\mathbf{E}})$ is of the form

$$
M(\widetilde{\mathbf{E}})=\left(\begin{array}{ccccccccc}
1 & \lambda & \lambda^{2} & \ldots & \lambda^{m} & y & * & \ldots & * \\
1 & \lambda & \lambda^{2} & \ldots & \lambda^{m} & z & * & \ldots & *
\end{array}\right) .
$$

As $p>3$, we can choose $y, z \in \mathbb{Z} / p \mathbb{Z}$ with $y \not \equiv z$ and $y, z \not \equiv \lambda^{m+1}(\bmod p)$ so that the condition in (3)(a) is satisfied.

Finally suppose that $\mathbf{a}=()$. Then

$$
M(\widetilde{\mathbf{E}})=\left(\begin{array}{cccccc}
1 & y & b_{1}+y c_{1} & * & \ldots & * \\
1 & z & b_{1}+z c_{1} & * & \ldots & *
\end{array}\right)
$$

for suitable $b_{1}, c_{1} \in \mathbb{Z} / p \mathbb{Z}$. We can choose $y, z \in \mathbb{Z} / p \mathbb{Z}$ with $y \not \equiv z$ such that

$$
y^{2} \not \equiv b_{1}+y c_{1} \quad \text { and } \quad z^{2} \not \equiv b_{1}+z c_{1} \quad(\bmod p),
$$

because quadratic equations have at most two solutions and $p>3$. Once more, the condition in (3)(a) is fulfilled.

The next result mimics [4, Lemma 3.2], which applies to GGS-groups. We remark that there are no new exceptions, in addition to the GGS-group

$$
\mathcal{G}=\langle a, b\rangle \quad \text { with } \quad \psi_{1}(b)=(a, a, \ldots, a, b),
$$

arising from a constant defining vector $(1, \ldots, 1)$. 
Proposition 3.5. Let $G=\left\langle a, b_{1}, \ldots, b_{r}\right\rangle$ be a multi-edge spinal group that is not conjugate to $\mathcal{G}$ in $\operatorname{Aut}(T)$. Then

$$
\psi_{1}\left(\gamma_{3}\left(\operatorname{Stab}_{G}(1)\right)\right)=\gamma_{3}(G) \times \stackrel{p}{.} \times \gamma_{3}(G) .
$$

In particular,

$$
\gamma_{3}(G) \times \stackrel{p}{.} \times \gamma_{3}(G) \subseteq \psi_{1}\left(\gamma_{3}(G)\right) .
$$

Proof. From $\psi_{1}\left(\operatorname{Stab}_{G}(1)\right) \subseteq G \times . \stackrel{p}{.} \times G$, we deduce that $\psi_{1}\left(\gamma_{3}\left(\operatorname{Stab}_{G}(1)\right)\right) \subseteq$ $\gamma_{3}(G) \times \stackrel{p}{.} \times \gamma_{3}(G)$, and so it suffices to prove the reverse inclusion. For $r=1$, the result has been proved in [4, Lemma 3.2]. Hence suppose that $r \geq 2$. For convenience, we prove the result in the isomorphic setting given by Lemma 3.4. That is, since

$$
\psi_{1}\left(\gamma_{3}\left(\operatorname{Stab}_{G}(1)\right)\right)^{f}=\psi_{1}\left(\gamma_{3}\left(\operatorname{Stab}_{G}(1)\right)^{f}\right)=\psi_{1}\left(\gamma_{3}\left(\operatorname{Stab}_{G^{f}}(1)\right)\right)
$$

and

$$
\left(\gamma_{3}(G) \times \ldots \times \gamma_{3}(G)\right)^{f}=\gamma_{3}\left(G^{f}\right) \times \ldots \times \gamma_{3}\left(G^{f}\right),
$$

for $f$ as in Lemma 3.4 , we may assume that $\psi_{1}\left(b_{i}\right)=\left(a^{e_{i, 1}}, \ldots, a^{e_{i, p-1}}, b_{i}\right)$ with $e_{i, 1}=1$, for $i \in\{1, \ldots, r\}$, and that the additional assertions (2), (3), (4) of Lemma 3.4 hold. Moreover, by Proposition 3.1 the group $G$ acts spherically transitively, hence it suffices to show that

$$
\gamma_{3}(G) \times 1 \times \ldots \times 1 \subseteq \psi_{1}\left(\gamma_{3}\left(\operatorname{Stab}_{G}(1)\right) .\right.
$$

We divide the argument into two cases.

Case 1: $(r, p) \neq(2,3)$. By Proposition 3.1, the group $G$ is fractal. Since $\overline{\gamma_{3}(G)}=\left\langle\left[a, b_{i}, a\right],\left[a, b_{i}, b_{j}\right] \mid 1 \leq i, j \leq r\right\rangle^{G}$, it suffices to construct elements in $\gamma_{3}\left(\operatorname{Stab}_{G}(1)\right)$ whose images under $\psi_{1}$ yield

$$
\left(\left[a, b_{i}, a\right], 1, \ldots, 1\right) \quad \text { and } \quad\left(\left[a, b_{i}, b_{j}\right], 1, \ldots, 1\right), \quad 1 \leq i, j \leq r .
$$

First suppose that $i \in\{1, \ldots, r\}$ with $e_{i, p-1} \equiv 0(\bmod p)$. Then

$$
\psi_{1}\left(b_{i}\right)=\left(a, a^{e_{i, 2}}, \ldots, a^{e_{i, p-2}}, 1, b_{i}\right) .
$$

Noting that $\left[b_{i}, b_{j}^{a}\right]=(*, 1, \ldots, 1, *)$ for $j \in\{1, \ldots, r\}$, where the symbols $*$ denote unspecified entries and the second $*$ equals 0 if $j=i$, we deduce that

$$
\begin{aligned}
\psi_{1}\left(\left[b_{i}, b_{i}^{a}, b_{i}\right]\right) & =\left(\left[a, b_{i}, a\right], 1, \ldots, 1\right), \\
\psi_{1}\left(\left[b_{i}, b_{i}^{a}, b_{i}^{a}\right]\right) & =\left(\left[a, b_{i}, b_{i}\right], 1, \ldots, 1\right),
\end{aligned}
$$

and for $j \in\{1, \ldots, r\}$ with $j \neq i$,

$$
\begin{aligned}
& \psi_{1}\left(\left[b_{i}, b_{i}^{a}, b_{j}^{a}\right]\right)=\left(\left[a, b_{i}, b_{j}\right], 1, \ldots, 1\right), \\
& \psi_{1}\left(\left[b_{i}, b_{j}^{a}, b_{i}^{a}\right]\right)=\left(\left[a, b_{j}, b_{i}\right], 1, \ldots, 1\right) .
\end{aligned}
$$

Next suppose that $i \in\{1, \ldots, r\}$ with $e_{i, p-1} \not \equiv 0(\bmod p)$. By properties (3) and (4) in Lemma 3.4 there exists $k \in\{2, \ldots, p-2\}$ such that $e_{i, k-1} e_{i, k+1} \not \equiv e_{i, k}^{2}(\bmod p)$, apart from an exceptional case which only occurs for $r=2$ and which we deal with separately below. Set

$$
g_{i, k}=\left(b_{i}^{a^{p-k+1}}\right)^{e_{i, k}}\left(b_{i}^{a^{p-k}}\right)^{-e_{i, k-1}}
$$


so that

$$
\psi_{1}\left(g_{i, k}\right)=\left(a^{e_{i, k}^{2}-e_{i, k-1} e_{i, k+1}}, *, \ldots, *, 1\right) .
$$

Since $e_{i, k}^{2}-e_{i, k-1} e_{i, k+1} \not \equiv 0(\bmod p)$, there is a power $g_{i}$ of $g_{i, k}$ such that

$$
\psi_{1}\left(g_{i}\right)=(a, *, \ldots, *, 1) .
$$

Additionally, since

$$
\psi_{1}\left(b_{i}^{a}\left(b_{i}^{a^{p-1}}\right)^{-e_{i, p-1}}\right)=\left(b_{i} a^{-e_{i, 2} e_{i, p-1}}, *, \ldots, *, 1\right),
$$

with the help of $g_{i}$ we get an element $h_{i} \in \operatorname{Stab}_{G}(1)$ such that

$$
\psi_{1}\left(h_{i}\right)=\left(b_{i}, *, \ldots, *, 1\right) .
$$

Consequently, we obtain

$$
\begin{aligned}
\psi_{1}\left(\left[b_{i}, b_{i}^{a}, g_{i}\right]\right) & =\left(\left[a, b_{i}, a\right], 1, \ldots, 1\right) \\
\psi_{1}\left(\left[b_{i}, b_{i}^{a}, h_{i}\right]\right) & =\left(\left[a, b_{i}, b_{i}\right], 1, \ldots, 1\right),
\end{aligned}
$$

and for $j \in\{1, \ldots, r\}$ with $j \neq i$,

$$
\psi_{1}\left(\left[b_{i}, b_{j}^{a}, h_{i}\right]\right)=\left(\left[a, b_{j}, b_{i}\right], 1, \ldots, 1\right)
$$

and

$\psi_{1}\left(\left[b_{i}, b_{i}^{a}, h_{j}\right]\right)=\left(\left[a, b_{i}, b_{j}\right], 1, \ldots, 1\right)$ if $e_{j, p-1} \not \equiv 0$,

$\psi_{1}\left(\left[b_{i}, b_{i}^{a}, b_{j}^{a}\right]\right)=\left(\left[a, b_{i}, b_{j}\right], 1, \ldots, 1\right)$ if $e_{j, p-1} \equiv 0$ (as in the previous part).

Thus we have constructed all necessary elements.

It remains to deal with the exceptional case which occurs only for $r=2$, and hence $p>3$. According to property (3b) in Lemma 3.4 we have

$$
e_{1}=(1,0, \ldots, 0), \quad e_{2}=(1,0, \ldots, 0,1),
$$

so that

$$
b_{1}=\left(a, 1, \ldots, 1,1, b_{1}\right), \quad b_{2}=\left(a, 1, \ldots, 1, a, b_{2}\right) .
$$

We simply replace $g_{2}$ in the above argument by $b_{2}^{a^{2}}=\left(a, b_{2}, a, 1, \ldots, 1\right)$, and then proceed similarly.

Case 2: $(r, p)=(2,3)$. Here $G^{\prime}=\gamma_{2}(G)=\left\langle\left[a, b_{1}\right],\left[a, b_{2}\right]\right\rangle^{G}$ and as $\left\langle a b_{2}, b_{2} a^{-1}\right\rangle=$ $\left\langle b_{2}^{2}, b_{2} a^{-1}\right\rangle=\left\langle a, b_{2}\right\rangle$, we have

$$
\gamma_{3}(G)=\left\langle\left[a, b_{1}, a\right],\left[a, b_{1}, b_{1}\right],\left[a, b_{1}, b_{2}\right],\left[a, b_{2}, b_{1}\right],\left[a, b_{2}, a b_{2}\right],\left[a, b_{2}, b_{2} a^{-1}\right]\right\rangle^{G} .
$$

By property (2) in Lemma 3.4 we may assume $\mathbf{e}_{1}=(1,0)$ and $\mathbf{e}_{2}=(1,-1)$ so that

$$
\psi_{1}\left(b_{1}\right)=\left(a, 1, b_{1}\right) \quad \text { and } \quad \psi_{1}\left(b_{2}\right)=\left(a, a^{-1}, b_{2}\right) .
$$

Arguing as in the previous case, it suffices to manufacture, in addition to the elements already constructed there, elements of $\gamma_{3}\left(\operatorname{Stab}_{G}(1)\right)$ whose images under $\psi_{1}$ are $\left(\left[a, b_{2}, a b_{2}\right], 1,1\right)$ and $\left(\left[a, b_{2}, b_{2} a^{-1}\right], 1,1\right)$. We compute

$$
\psi_{1}\left(b_{2} b_{2}^{a}\right)=\left(a b_{2}, 1, b_{2} a^{-1}\right) \quad \text { and } \quad \psi_{1}\left(b_{2}^{a} b_{2}^{a^{2}}\right)=\left(b_{2} a^{-1}, a b_{2}, 1\right) \text {, }
$$


yielding

$$
\begin{aligned}
\psi_{1}\left(\left[b_{2}, b_{2}^{a}, b_{2}^{a} b_{2}^{a^{2}}\right]\right) & =\left(\left[a, b_{2}, b_{2} a^{-1}\right], 1,1\right), \\
\psi_{1}\left(\left[b_{2}^{-a^{2}}, b_{2}^{a}, b_{2} b_{2}^{a}\right]\right) & =\left(\left[a, b_{2}, a b_{2}\right], 1,1\right) .
\end{aligned}
$$

The following consequence paves the way to proving branchness.

Proposition 3.6. Let $G$ be a multi-edge spinal group that is not $\operatorname{Aut}(T)$ conjugate to the $G G S$-group $\mathcal{G}$ in (3.7). Then $\gamma_{3}(G) \subseteq \operatorname{Rstab}_{G}(u)_{u}$ for every vertex $u$ of $T$, after the natural identification of subtrees.

Proof. Let $u$ be a vertex of $T$ at level $n$. We induct on $n$. If $n=0$ then $u=\varnothing$ is the root vertex and the claim holds trivially. Now suppose that $n>0$. Writing $u$ as $u=v y$, where $v$ is a vertex at level $n-1$ and $y \in X$, we conclude by induction that $\gamma_{3}(G) \subseteq \operatorname{Rstab}_{G}(v)_{v}$. By Proposition 3.5.

$$
\gamma_{3}(G) \times \stackrel{p}{.} \times \gamma_{3}(G) \subseteq \psi_{1}\left(\gamma_{3}(G)\right) \subseteq \psi_{1}\left(\operatorname{Rstab}_{G}(v)_{v}\right)
$$

so that, in particular,

$$
1 \times \ldots \times 1 \times \gamma_{3}(G) \times 1 \times \ldots \times 1 \subseteq \psi_{1}\left(\operatorname{Rstab}_{G}(v)_{v}\right),
$$

where $\gamma_{3}(G)$ is located at position $u$ in the subtree $T_{v}$ rooted at $v$. Hence $\gamma_{3}(G) \subseteq \operatorname{Rstab}_{G}(u)_{u}$.

Proposition 3.7. Let $G$ be a multi-edge spinal group that is not $\operatorname{Aut}(T)$ conjugate to the GGS-group $\mathcal{G}$ in (3.7). Then $G$ is a branch group.

Proof. In view of Proposition 3.1 it suffices to show that every rigid level stabilizer $\operatorname{Rstab}_{G}(n)$ is of finite index in $G$. The nilpotent quotient $G / \gamma_{3}(G)$ is generated by finitely many elements of finite order and hence finite. Thus $\gamma_{3}(G)$ has finite index in $G$. By Proposition 3.6, the image of $\operatorname{Rstab}_{G}(n)$ under the maps $\psi_{n}$ contains the direct product of $p^{n}$ copies of $\gamma_{3}(G)$. Since the image of any level stabilizer $\operatorname{Stab}_{G}(n)$ under the injective map $\psi_{n}$ is contained in the direct product of $p^{n}$ copies of $G$, we deduce that $\operatorname{Rstab}_{G}(n)$ is of finite index in $\operatorname{Stab}_{G}(n)$ and hence in $G$.

Theorem 3.8 ([6, Theorem 4]). A branch group $G$ with branch structure $\left(\left(L_{n}\right)_{n=1}^{\infty},\left(H_{n}\right)_{n=1}^{\infty}\right)$ is just infinite if and only if for each $n \geq 1$, the index of the commutator subgroup $L_{n}^{\prime}$ in $L_{n}$ is finite.

Corollary 3.9 ([6, Section 7]). Every finitely generated, torsion branch group $G$ is just infinite.

Proof. As $G$ is branch, $L_{n}=L_{n}^{G}$ is of finite index in $G$. Hence $L_{n}$ is a finitely generated torsion group and $L_{n} / L_{n}^{\prime}$ is finite abelian.

We do not know a proof that the GGS-group $\mathcal{G}$ in (3.7) is not branch. From properties that were established in [4] we derive the following result.

Proposition 3.10. The GGS-group $\mathcal{G}$ in (3.7) is not just infinite. 
Proof. Write $G=\mathcal{G}=\langle a, b\rangle$ with $\psi_{1}(b)=(a, \ldots, a, b)$, and put $K=$ $\left\langle b a^{-1}\right\rangle^{G}$. From [4, Section 4] we have that

(1) $|G: K|=p$ and $K^{\prime}=\left\langle\left[\left(b a^{-1}\right)^{a}, b a^{-1}\right]\right\rangle^{G} \leq \operatorname{Stab}_{G}(1)$;

(2) $\left|G / K^{\prime} \operatorname{Stab}_{G}(n)\right|=p^{n+1}$ for every $n \in \mathbb{N}$ with $n \geq 2$.

Hence $K^{\prime}$ is a non-trivial normal subgroup of infinite index in $G$.

What about just infinite multi-edge spinal groups that are not torsion? For $p \geq 5$, it is shown in [6, Example 7.1] that the non-torsion group $G=$ $\langle a, b\rangle$ with $\psi_{1}(b)=(a, 1, \ldots, 1, b)$ is just infinite, and more generally in [6, Example 10.2] that $G=\langle a, b\rangle$ with $\psi_{1}(b)=\left(a^{e_{1}}, a^{e_{2}}, \ldots, a^{e_{p-4}}, 1,1,1, b\right)$ where $e_{1} \not \equiv 0$ is just infinite. For the latter example, when $\sum_{i=1}^{p-4} e_{i} \not \equiv 0$ $(\bmod p)$, then the group is non-torsion.

Now let $G$ be the multi-edge spinal group with defining vectors $\mathbf{e}_{i}$ of the form $\left(e_{i, 1}, e_{i, 2}, \ldots, e_{i, p-2}, e_{i, p-1}\right)$ satisfying $e_{i, 1} \not \equiv 0(\bmod p)$ and $e_{i, p-3} \equiv$ $e_{i, p-2} \equiv e_{i, p-1} \equiv 0(\bmod p)$ for every $i \in\{1, \ldots, r\}$. As in [6, Example 10.2], it can be shown that $G$ is just infinite, and furthermore when $\sum_{j=1}^{p-4} e_{i, j} \not \equiv 0$ $(\bmod p)$ for at least one $i \in\{1, \ldots, r\}$, then $G$ is non-torsion. It is not always the case that the last three entries of the defining vectors are to be zero. For example, the non-torsion multi-edge spinal group $G$ with $e_{i, 1} \equiv$ $e_{i, p-2} \equiv e_{i, p-1} \equiv 0(\bmod p)$ and $e_{i, 2} \not \equiv 0(\bmod p)$ is likewise just infinite.

\section{THETA MAPS}

Here we determine the abelianization $G / G^{\prime}$ of a multi-edge spinal group $G$. Then we define a natural length function on elements of the commutator subgroup $G^{\prime}$. Akin to Pervova's work [12, we introduce two theta maps $\Theta_{1}, \Theta_{2}: G^{\prime} \rightarrow G^{\prime}$ which are key to establishing that all maximal subgroups of $G$ are of finite index. We prove that the length of every element of the commutator subgroup of length at least 3 decreases under repeated applications of a combination of these maps. Our use of two theta maps, instead of one as in [12] allows us to significantly simplify the calculations.

4.1. Abelianization of multi-edge spinal groups. Recall that every element $g$ of the free product $\operatorname{Fr}_{\lambda \in \Lambda} \Gamma_{\lambda}$ of a family of groups $\left(\Gamma_{\lambda}\right)_{\lambda \in \Lambda}$ can be uniquely represented as a reduced word in $\sqcup_{\lambda \in \Lambda} \Gamma_{\lambda}$, i.e., a word $g=$ $g_{1} g_{2} \cdots g_{n}$, where $n \in \mathbb{N} \cup\{0\}, \lambda_{1}, \ldots, \lambda_{n} \in \Lambda$ with $\lambda_{i} \neq \lambda_{i+1}$ for $1 \leq i \leq$ $n-1$, and $1 \neq g_{i} \in \Gamma_{\lambda_{i}}$ for each $i \in\{1, \ldots, n\}$.

Let $G=G_{\mathbf{E}}=\left\langle a, b_{1}, \ldots, b_{r}\right\rangle$ be a multi-edge spinal group acting on the regular $p$-adic rooted tree $T$, for an odd prime $p$. Here $\mathbf{E}$ is the $r$-tuple of defining vectors $\mathbf{e}_{i}=\left(e_{i, 1}, \ldots, e_{i, p-1}\right)$, for $i \in\{1, \ldots, r\}$.

In order to study $G / G^{\prime}$ we consider

$$
\begin{aligned}
& H=\left\langle\hat{a}, \hat{b}_{1}, \ldots, \hat{b}_{r}\right| \\
& \left.\quad \hat{a}^{p}=\hat{b}_{1}^{p}=\ldots=\hat{b}_{r}^{p}=1, \text { and }\left[\hat{b}_{i}, \hat{b}_{j}\right]=1 \text { for } 1 \leq i, j \leq r\right\rangle,
\end{aligned}
$$


the free product $\langle\hat{a}\rangle *\left\langle\hat{b}_{1}, \ldots, \hat{b}_{r}\right\rangle$ of a cyclic group $\langle\hat{a}\rangle \cong C_{p}$ and an elementary abelian group $\left\langle\hat{b}_{1}, \ldots, \hat{b}_{r}\right\rangle \cong C_{p}^{r}$. There is a unique epimorphism $\pi: H \rightarrow G$ such that $\hat{a} \mapsto a$ and $\hat{b}_{i} \mapsto b_{i}$ for $i \in\{1, \ldots, r\}$, inducing an epimorphism from $H / H^{\prime} \cong\langle\hat{a}\rangle \times\left\langle\hat{b}_{1}, \ldots, \hat{b}_{r}\right\rangle \cong C_{p}^{r+1}$ onto $G / G^{\prime}$. We want to show that the latter is an isomorphism; see Proposition 4.3 below.

Let $h \in H$. As discussed, each $h$ can be uniquely represented in the form

$$
h=\hat{a}^{s_{1}} \cdot\left(\hat{b}_{1}^{\beta_{1,1}} \ldots \hat{b}_{r}^{\beta_{r, 1}}\right) \cdot \hat{a}^{s_{2}} \cdot \ldots \cdot \hat{a}^{s_{m}} \cdot\left(\hat{b}_{1}^{\beta_{1, m}} \ldots \hat{b}_{r}^{\beta_{r, m}}\right) \cdot \hat{a}^{s_{m+1}},
$$

where $m \in \mathbb{N} \cup\{0\}$ and $s_{1}, \ldots, s_{m+1}, \beta_{1,1}, \ldots, \beta_{r, m} \in \mathbb{Z} / p \mathbb{Z}$ with

$$
s_{i} \not \equiv 0 \quad(\bmod p) \quad \text { for } i \in\{2, \ldots, m\},
$$

and for each $j \in\{1, \ldots, m\}$,

$$
\beta_{i, j} \not \equiv 0 \quad(\bmod p) \quad \text { for at least one } i \in\{1, \ldots, r\} .
$$

We denote by $\partial(h)=m$ the length of $h$, with respect to the factor $\left\langle\hat{b}_{1}, \ldots, \hat{b}_{r}\right\rangle$. Clearly, for $h_{1}, h_{2} \in H$ we have

$$
\partial\left(h_{1} h_{2}\right) \leq \partial\left(h_{1}\right)+\partial\left(h_{2}\right) .
$$

In addition, we define exponent maps

$$
\begin{aligned}
\varepsilon_{\hat{a}}(h) & =\sum_{j=1}^{m+1} s_{j} \in \mathbb{Z} / p \mathbb{Z} \quad \text { and } \\
\varepsilon_{\hat{b}_{i}}(h) & =\sum_{j=1}^{m} \beta_{i, j} \in \mathbb{Z} / p \mathbb{Z} \quad \text { for } i \in\{1, \ldots, r\}
\end{aligned}
$$

with respect to the generating set $\hat{a}, \hat{b}_{1}, \ldots, \hat{b}_{r}$.

The surjective homomorphism

$$
H \rightarrow(\mathbb{Z} / p \mathbb{Z}) \times(\mathbb{Z} / p \mathbb{Z})^{r}, \quad h \mapsto\left(\varepsilon_{\hat{a}}(h), \varepsilon_{\hat{b}_{1}}(h), \ldots, \varepsilon_{\hat{b}_{r}}(h)\right)
$$

has kernel $H^{\prime}$ and provides an explicit model for the abelianization $H / H^{\prime}$. The group $L(H)=\left\langle\hat{b}_{1}, \ldots, \hat{b}_{r}\right\rangle^{H}$ is the kernel of the surjective homomorphism

$$
H \rightarrow \mathbb{Z} / p \mathbb{Z}, \quad h \mapsto \varepsilon_{\hat{a}}(h) .
$$

Each element $h \in L(H)$ can be uniquely represented by a word of the form

$$
h=\left(\hat{c}_{1}\right)^{\hat{a}^{t_{1}}} \cdots\left(\hat{c}_{m}\right)^{\hat{a}^{t_{m}}},
$$

where $m \in \mathbb{N} \cup\{0\}$ and $t_{1}, \ldots, t_{m} \in \mathbb{Z} / p \mathbb{Z}$ with $t_{j} \not \equiv t_{j+1}(\bmod p)$ for $j \in\{1, \ldots, m-1\}$, and for each $j \in\{1, \ldots, m\}$,

$$
\hat{c}_{j}=\hat{b}_{1}^{\beta_{1, j}} \ldots \hat{b}_{r}^{\beta_{r, j}} \in\left\langle\hat{b}_{1}, \ldots, \hat{b}_{r}\right\rangle \backslash\{1\} .
$$

Let $\alpha$ denote the cyclic permutation of the factors of $H \times \stackrel{p}{.} \times H$ corresponding to the $p$-cycle $(12 \ldots p)$. We consider the homomorphism

$$
\Phi: L(H) \rightarrow H \times \stackrel{p}{ } . \times H
$$

defined by

$$
\Phi\left(\hat{b}_{i}^{\hat{a}^{k}}\right)=\left(\hat{a}^{e_{i, 1}}, \ldots, \hat{a}^{e_{i, p-1}}, \hat{b}_{i}\right)^{\alpha^{k}} \quad \text { for } i \in\{1, \ldots, r\}, k \in \mathbb{Z} / p \mathbb{Z} .
$$


Lemma 4.1. Let $H$ be as above, and $h \in L(H)$ with $\Phi(h)=\left(h_{1}, \ldots, h_{p}\right)$. Then $\sum_{i=1}^{p} \partial\left(h_{i}\right) \leq \partial(h)$, and $\partial\left(h_{i}\right) \leq\left\lceil\frac{\partial(h)}{2}\right\rceil$ for each $i \in\{1, \ldots, p\}$.

Proof. Suppose that $h$ is of length $\partial(h)=m$ as in (4.6). For each $j \in$ $\{1, \ldots, m\}$ the factor $\left(\hat{c}_{j}\right)^{\hat{a}^{t_{j}}}$ in 4.6 contributes to precisely one coordinate of $\Phi(h)$ a factor $\hat{c}_{j}$ and to all other coordinates a power of $\hat{a}$. Therefore $\sum_{i=1}^{p} \partial\left(h_{i}\right) \leq m$.

Now let $i \in\{1, \ldots, p\}$. The maximum length in the $i$ th coordinate occurs when $h_{i}$ is of the form $\hat{c}_{*} \hat{a}^{*} \hat{c}_{*} \ldots \hat{a}^{*} \hat{c}_{*}$ with $m$ factors, where the symbols $*$ represent suitable indices or exponents. Therefore $\partial\left(h_{i}\right) \leq\lceil m / 2\rceil$.

The following proposition provides a recursive presentation for a multiedge spinal group. It can be extracted from a result of Rozhkov [13; a self-contained proof for multi-edge spinal groups is included in [1].

Proposition 4.2. Let $G=\left\langle a, b_{1}, \ldots, b_{r}\right\rangle$ be a multi-edge spinal group, and $H$ as in (4.1). Consider the subgroup $K=\bigcup_{n=0}^{\infty} K_{n}$ of $H$, where

$$
K_{0}=\{1\} \quad \text { and } \quad K_{n}=\Phi^{-1}\left(K_{n-1} \times \ldots \times K_{n-1}\right) \text { for } n \geq 1 .
$$

Then $K \subseteq L(H)=\left\langle\hat{b}_{1}, \ldots, \hat{b}_{r}\right\rangle^{H}$, and $K$ is normal in $H$. Moreover, the epimorphism $\pi: H \rightarrow G$ given by $\hat{a} \mapsto a, \hat{b}_{i} \mapsto b_{i}$, for $i \in\{1, \ldots, r\}$, has $\operatorname{ker}(\pi)=K$. In particular, $G \cong H / K$.

Next we describe the abelianization of a multi-edge spinal group.

Proposition 4.3. Let $G=\left\langle a, b_{1}, \ldots, b_{r}\right\rangle$ be a multi-edge spinal group, and $H$ as in 4.1. Then the map $H \rightarrow(\mathbb{Z} / p \mathbb{Z}) \times(\mathbb{Z} / p \mathbb{Z})^{r}$ in (4.5) factors through $G / G^{\prime}$. Consequently,

$$
G / G^{\prime} \cong H / H^{\prime} \cong C_{p}^{r+1}
$$

Proof. Below we prove that

$$
\Phi^{-1}\left(H^{\prime} \times \stackrel{p}{.} . \times H^{\prime}\right) \leq H^{\prime} .
$$

Let $K=\bigcup_{n=0}^{\infty} K_{n} \leq L(H)$ be as in Proposition 4.2 so that the natural epimorphism $\pi: H \rightarrow G$ has $\operatorname{ker}(\pi)=K$, and $G \cong H / K$. From (4.8), we deduce by induction that $K_{n} \leq H^{\prime}$ for all $n \in \mathbb{N} \cup\{0\}$, hence $K \leq H^{\prime}$ and $G / G^{\prime} \cong H / H^{\prime} K=H / H^{\prime}$.

It remains to justify (4.8). Consider an arbitrary element $h \in L(H)$ as in (4.6) and (4.7). We write $\Phi(h)=\left(h_{1}, \ldots, h_{p}\right)$. For $i \in\{1, \ldots, r\}$ and $k \in\{1, \ldots, p\}$, let $\varepsilon_{\hat{b}_{i}, k}(h)$ be the sum of exponents $\beta_{i, j}, j \in\{1, \ldots, m\}$ with $t_{j}=k$, so that $\varepsilon_{\hat{b}_{i}}\left(h_{k}\right)=\varepsilon_{\hat{b}_{i}, k}(h)$. It follows that for each $i \in\{1, \ldots, r\}$,

$$
\varepsilon_{\hat{b}_{i}}(h)=\sum_{j=1}^{m} \beta_{i, j}=\sum_{k=1}^{p} \varepsilon_{\hat{b}_{i}, k}(h)=\sum_{k=1}^{p} \varepsilon_{\hat{b}_{i}}\left(h_{k}\right) .
$$

Now suppose that $h \notin H^{\prime}$. From (4.5) and $\varepsilon_{\hat{a}}(H)=0$ we deduce that $\varepsilon_{\hat{b}_{i}}(h) \not \equiv 0$ for at least one $i \in\{1, \ldots, r\}$. Thus 4.9 implies that $\varepsilon_{\hat{b}_{i}}\left(h_{k}\right) \not \equiv 0$ for some $k \in\{1, \ldots, p\}$ and $\Phi(h) \notin H^{\prime} \times . \stackrel{p}{.} \times H^{\prime}$. Therefore 4.8 holds. 
As above, let $G=\left\langle a, b_{1}, \ldots, b_{r}\right\rangle$ be a multi-edge spinal group, and $\pi: H \rightarrow$ $G$ the natural epimorphism with $H$ as in (4.1). The length of $g \in G$ is

$$
\partial(g)=\min \left\{\partial(h) \mid h \in \pi^{-1}(g)\right\} .
$$

Based on (4.3), one easily shows that for $g_{1}, g_{2} \in G$,

$$
\partial\left(g_{1} g_{2}\right) \leq \partial\left(g_{1}\right)+\partial\left(g_{2}\right) .
$$

Moreover, using Proposition 4.3 we may define $\varepsilon_{a}(g), \varepsilon_{b_{1}}(g), \ldots, \varepsilon_{b_{r}}(g) \in$ $\mathbb{Z} / p \mathbb{Z}$ via any pre-image $h \in \pi^{-1}(g)$ :

$$
\left(\varepsilon_{a}(g), \varepsilon_{b_{1}}(g), \ldots, \varepsilon_{b_{r}}(g)\right)=\left(\varepsilon_{\hat{a}}(h), \varepsilon_{\hat{b}_{1}}(h), \ldots, \varepsilon_{\hat{b}_{r}}(h)\right) .
$$

We record the following direct consequences of Lemma 4.1

Lemma 4.4. Let $G$ be a multi-edge spinal group as above, and $g \in \operatorname{Stab}_{G}(1)$ with $\psi_{1}(g)=\left(g_{1}, \ldots, g_{p}\right)$. Then $\sum_{i=1}^{p} \partial\left(g_{i}\right) \leq \partial(g)$, and $\partial\left(g_{i}\right) \leq\left\lceil\frac{\partial(g)}{2}\right\rceil$ for each $i \in\{1, \ldots, p\}$.

In particular, if $\partial(g)>1$ then $\partial\left(g_{i}\right)<\partial(g)$ for every $i \in\{1, \ldots, p\}$.

4.2. Length reduction. We continue to consider a multi-edge spinal group $G=G_{\mathbf{E}}=\left\langle a, b_{1}, \ldots, b_{r}\right\rangle$ acting on the regular $p$-adic rooted tree $T$, for an odd prime $p$. Here $\mathbf{E}$ is the $r$-tuple of defining vectors $\mathbf{e}_{i}=\left(e_{i, 1}, \ldots, e_{i, p-1}\right)$, for $i \in\{1, \ldots, r\}$. In preparation for the investigation of maximal subgroups of $G$, we introduce in the present section two length decreasing maps $\Theta_{1}, \Theta_{2}: G^{\prime} \rightarrow G^{\prime}$. Based on Lemma 3.3 , we assume that $e_{1,1}=1$. Also, let

$$
n=\max \left\{j \in\{1, \ldots, p-1\} \mid e_{1, j} \not \equiv 0 \quad(\bmod p)\right\} .
$$

Generically, we have $n \geq 2$, while the exceptional case $n=1$ corresponds to $b_{1}$ of the form $\psi_{1}\left(b_{1}\right)=\left(a, 1, \ldots, 1, b_{1}\right)$. The special case $n=1$ will be dealt with slightly differently in what follows, and it only applies to a specific family of groups. We remark that, if $G$ is torsion, then Theorem 3.2 automatically yields $n \geq 2$.

Clearly, $G^{\prime}=\left\langle\left[a, b_{i}\right] \mid i \in\{1, \ldots, r\}\right\rangle^{G}$ is a subgroup of $\operatorname{Stab}_{G}(1)=$ $\left\langle b_{1}, \ldots, b_{r}\right\rangle^{G}$. Every $g \in \operatorname{Stab}_{G}(1)$ has a decomposition

$$
\psi_{1}(g)=\left(g_{1}, \ldots, g_{p}\right)
$$

where each $g_{j} \in U_{u_{j}}^{G} \cong G$ is an element of the upper companion group acting on the subtree rooted at a first level vertex $u_{j}, j \in\{1, \ldots, p\}$, and we define

$$
\varphi_{j}: \operatorname{Stab}_{G}(1) \rightarrow \operatorname{Aut}\left(T_{u_{j}}\right), \quad \varphi_{j}(g)=g_{j} .
$$

It is customary and useful to write $\left(g_{1}, \ldots, g_{p}\right)$ in place of $g \in \operatorname{Stab}_{G}(1)$ to carry out certain computations.

We are interested in projecting, via $\varphi_{p}$, the first level stabilizer $\operatorname{Stab}_{M}(1)$ of a subgroup $M \leq G$, containing $b_{1}$ and an 'approximation' $a z \in a G^{\prime}$ of $a$, 
to a subgroup of $\operatorname{Aut}\left(T_{u_{p}}\right)$. Writing $\psi_{1}(z)=\left(z_{1}, \ldots, z_{p}\right)$ and conjugating $b_{1}$ by $(a z)^{-1}$, we obtain

$$
\begin{aligned}
b_{1}^{(a z)^{-1}} & =\left(z \cdot\left(a, a^{e_{1,2}}, \ldots, a^{e_{1, p-1}}, b_{1}\right) \cdot z^{-1}\right)^{a^{-1}} \\
& =\left(a^{z_{1}^{-1}},\left(a^{e_{1,2}}\right)^{z_{2}^{-1}}, \ldots,\left(a^{e_{1, p-1}}\right)^{z_{p-1}^{-1}}, b_{1}^{z_{p}^{-1}}\right)^{a^{-1}} \\
& =\left(\left(a^{e_{1,2}}\right)^{z_{2}^{-1}}, \ldots,\left(a^{e_{1, p-1}}\right)^{z_{p-1}^{-1}}, b_{1}^{z_{p}^{-1}}, a^{z_{1}^{-1}}\right) .
\end{aligned}
$$

Therefore

$$
\varphi_{p}\left(b_{1}^{(a z)^{-1}}\right)=a^{z_{1}^{-1}}=a\left[a, z_{1}^{-1}\right]
$$

and this motivates us to define

$$
\Theta_{1}: G^{\prime} \rightarrow G^{\prime}, \quad \Theta_{1}(z)=\left[a, z_{1}^{-1}\right] .
$$

The map $\Theta_{2}$ is obtained similarly. As $e_{1, n} \not \equiv 0$, we find $k \in \mathbb{Z} / p \mathbb{Z}$ such that $k e_{1, n} \equiv 1(\bmod p)$. Writing $\widetilde{e}_{1, j}=k e_{1, j}$ for $j \in\{1, \ldots, p-1\}$, we obtain by induction on $p-n$ that

$$
\begin{aligned}
\left(b_{1}^{k}\right)^{(a z)^{p-n}} & =\left(z^{-1} \cdot\left(a^{\widetilde{e}_{1,1}}, \ldots, a^{\widetilde{e}_{1, n-1}}, a, a^{\widetilde{e}_{1, n+1}}, \ldots, a^{\widetilde{e}_{1, p-1}}, b_{1}^{k}\right)^{a} \cdot z\right)^{(a z)^{p-n-1}} \\
& =\left(\left(b_{1}^{k}\right)^{z_{1}},\left(a^{\widetilde{e}_{1,1}}\right)^{z_{2}}, \ldots,\left(a^{\widetilde{e}_{1, n-1}}\right)^{z_{n}}, a^{z_{n+1}}, *, \ldots, *\right)^{(a z)^{p-n-1}} \\
& =\left(*, \ldots, *, a^{z_{n+1} z_{n+2} \cdots z_{p}}\right)
\end{aligned}
$$

where the symbols $*$ represent unspecified components. Therefore

$$
\varphi_{p}\left(\left(b_{1}^{k}\right)^{(a z)^{p-n}}\right)=a^{z_{n+1} \cdots z_{p}}=a\left[a, z_{n+1} \cdots z_{p}\right]
$$

and this motivates us to define

$$
\Theta_{2}: G^{\prime} \rightarrow G^{\prime}, \quad \Theta_{2}(z)=\left[a, z_{n+1} \ldots z_{p}\right] .
$$

To deal with the case $n=1$, we define $\mathcal{E}$ to be the family of all multi-edge spinal groups $G=\left\langle a, b_{1}, \ldots, b_{r}\right\rangle$ that satisfy

$$
\begin{cases}\psi_{1}\left(b_{1}\right)=\left(a, 1, \ldots, 1, b_{1}\right) & \\ e_{i, 1} \equiv 1 \quad(\bmod p) & \text { for every } i \in\{1, \ldots, r\} \\ e_{i, p-1} \neq \equiv 0 \quad(\bmod p) & \text { for at least one } i \in\{1, \ldots, r\} .\end{cases}
$$

We remark that, by Theorem 3.2 , there are no torsion groups in $\mathcal{E}$.

Theorem 4.5. Let $G=\left\langle a, b_{1}, \ldots, b_{r}\right\rangle$ be a multi-edge spinal group acting on the regular $p$-adic rooted tree $T$, for an odd prime $p$. Suppose $G$ is not $\operatorname{Aut}(T)$-conjugate to a group in $\mathcal{E}$. Then the length $\partial(z)$ of an element $z \in G^{\prime}$ decreases under repeated applications of a suitable combination of the maps $\Theta_{1}$ and $\Theta_{2}$ down to length 0 or 2 .

Proof. Let $z \in G^{\prime}$. We observe that $\partial(z) \neq 1$; see Proposition 4.3 . Suppose that $\partial(z)=m \geq 3$. Then $z \in G^{\prime} \subseteq \operatorname{Stab}_{G}(1)$ has a decomposition

$$
\psi_{1}(z)=\left(z_{1}, \ldots, z_{p}\right) .
$$

From Lemma 4.4 and 4.10 we obtain $\partial\left(z_{j}\right) \leq\left\lceil\frac{m}{2}\right\rceil$ for $j \in\{1, \ldots, p\}$ and

$$
\partial\left(z_{1}\right)+\partial\left(z_{n+1} \cdots z_{p}\right) \leq m .
$$


If $\partial\left(z_{1}\right)<\frac{m}{2}$ then $\partial\left(\Theta_{1}(z)\right)<m$, and likewise if $\partial\left(z_{n+1} \cdots z_{p}\right)<\frac{m}{2}$ then $\partial\left(\Theta_{2}(z)\right)<m$. Hence we may suppose that $m=2 \mu$ is even and

$$
\partial\left(z_{1}\right)=\partial\left(z_{n+1} \cdots z_{p}\right)=\mu \text {. }
$$

We write $z_{n+1} \cdots z_{p}$ as

$$
a^{s_{1}} \cdot c_{1} \cdot a^{s_{2}} \cdot \ldots \cdot a^{s_{\mu}} \cdot c_{\mu} \cdot a^{s_{\mu+1}},
$$

where $s_{1}, \ldots, s_{\mu+1} \in \mathbb{Z} / p \mathbb{Z}$ with $s_{i} \not \equiv 0(\bmod p)$ for $i \in\{2, \ldots, \mu\}$ and $c_{1}, \ldots, c_{\mu} \in\left\langle b_{1}, \ldots, b_{r}\right\rangle \backslash\{1\}$, and distinguish two cases. To increase the readability of exponents we use at times also the notation $s(i)=s_{i}$.

Case 1: $s_{\mu+1} \equiv 0(\bmod p)$. Expressing

$$
\Theta_{2}(z)=\left[a, z_{n+1} \cdots z_{p}\right]=\left[a, a^{s_{1}} c_{1} a^{s_{2}} \cdots a^{s_{\mu}} c_{\mu}\right]
$$

as a product of conjugates of the $c_{i}^{ \pm 1}$ by powers of $a$ and relabelling the $c_{i}^{ \pm 1}$ as $\bar{c}_{j}$ for $j \in\{1, \ldots, m\}$, we get

$$
\Theta_{2}(z)=\bar{c}_{1}^{a} \cdot \bar{c}_{2}^{a^{1+s(\mu)}} \cdot \ldots \cdot \bar{c}_{\mu}^{a^{1+s(\mu)+\ldots+s(2)}} \cdot \bar{c}_{\mu+1}^{a^{s(\mu)+\ldots+s(2)}} \cdot \ldots \cdot \bar{c}_{m-1}^{a^{s(\mu)}} \cdot \bar{c}_{m} .
$$

Consider now

$$
\psi_{1}\left(\Theta_{2}(z)\right)=\left(\left(\Theta_{2}(z)\right)_{1}, \ldots,\left(\Theta_{2}(z)\right)_{p}\right) .
$$

If $\partial\left(\left(\Theta_{2}(z)\right)_{1}\right)<\mu$ then $\Theta_{1}\left(\Theta_{2}(z)\right)$ has length less than $m$. Hence we suppose

$$
\partial\left(\left(\Theta_{2}(z)\right)_{1}\right)=\mu \text {. }
$$

Using the symbol $*$ for unspecified exponents, we deduce from (4.14) that the first components $\left(\bar{c}_{j}^{a^{*}}\right)_{1}$ for odd $j \in\{1, \ldots, m-1\}$ must be non-trivial elements of $\left\langle b_{1}, \ldots, b_{r}\right\rangle$, and the $\left(\bar{c}_{j}^{a^{*}}\right)_{1}$ for even $j \in\{2, \ldots, m-2\}$ must be non-trivial powers of $a$. In particular, looking at the $(m-1)$ th term we require $s_{\mu} \equiv 1(\bmod p)$. This implies that the second factor in 4.14$)$ is $\bar{c}_{2}^{a^{2}}$.

In the special case $n=1, e_{i, p-1} \equiv 0(\bmod p)$ for every $i \in\{1, \ldots, r\}$ and so we immediately get a contradiction, because $\left(\bar{c}_{2}^{a^{2}}\right)_{1}$ contributes a trivial factor 1 to $\left(\Theta_{2}(z)\right)_{1}$ instead of a non-trivial power of $a$.

In the generic case $n \geq 2$ we claim $\partial\left(\left(\Theta_{2}(z)\right)_{n+1} \cdots\left(\Theta_{2}(z)\right)_{p}\right)<\mu$, leading to $\partial\left(\Theta_{2}\left(\Theta_{2}(z)\right)\right)<m$. Indeed, only factors $\bar{c}_{j}^{a^{*}}$ in 4.14 for even $j \in$ $\{2, \ldots, m\}$ can contribute non-trivial elements of $\left\langle b_{1}, \ldots, b_{r}\right\rangle$ to the product $\left(\Theta_{2}(z)\right)_{n+1} \cdots\left(\Theta_{2}(z)\right)_{p}$. But since $n \geq 2$, the second factor $\bar{c}_{2}^{a^{2}}$ in 4.14 contributes only a power of $a$.

Case 2: $s_{\mu+1} \not \equiv 0(\bmod p)$. Similarly as in Case 1 , we write

$$
\Theta_{2}(z)=\bar{c}_{1}^{a^{1+s(\mu+1)}} \cdot \bar{c}_{2}^{a^{1+s(\mu+1)+s(\mu)}} \cdot \ldots \cdot \bar{c}_{m-1}^{a^{s(\mu+1)+s(\mu)}} \cdot \bar{c}_{m}^{a^{s(\mu+1)}},
$$

where the $c_{i}^{ \pm 1}$ are relabelled as $\bar{c}_{j}$ for $j \in\{1, \ldots, m\}$. As before, it suffices to show that $\partial\left(\left(\Theta_{2}(z)\right)_{1}\right)<\mu$ or $\partial\left(\left(\Theta_{2}(z)\right)_{n+1} \cdots\left(\Theta_{2}(z)\right)_{p}\right)<\mu$.

Suppose $\partial\left(\left(\Theta_{2}(z)\right)_{1}\right)=\mu$. Then either:

(i) $\left(\bar{c}_{j}^{a^{*}}\right)_{1}$ for odd $j \in\{1, \ldots, m-1\}$ is a non-trivial element of $\left\langle b_{1}, \ldots, b_{r}\right\rangle$, and $\left(\bar{c}_{j}^{a^{*}}\right)_{1}$ for even $j \in\{2, \ldots, m-2\}$ is a non-trivial power of $a$; or 
(ii) $\left(\bar{c}_{j}^{a^{*}}\right)_{1}$ for even $j \in\{2, \ldots, m\}$ is a non-trivial element of $\left\langle b_{1}, \ldots, b_{r}\right\rangle$, and $\left(\bar{c}_{j}^{a^{*}}\right)_{1}$ for odd $j \in\{3, \ldots, m-1\}$ is a non-trivial power of $a$.

In case $(\mathrm{i})$, we deduce from $(m-1)$ th term in 4.15 that

$$
s_{\mu+1}+s_{\mu} \equiv 1 \quad(\bmod p),
$$

and the second term in 4.15 is equal to $\bar{c}_{2}^{a^{2}}$. We may argue as in Case 1 that $\partial\left(\left(\Theta_{2}(z)\right)_{n+1} \cdots\left(\Theta_{2}(z)\right)_{p}\right)<\mu$ so that $\partial\left(\Theta_{2}\left(\Theta_{2}(z)\right)\right)<m$.

In case (ii), we deduce from the $m$ th term in 4.15 that

$$
s_{\mu+1} \equiv 1 \quad(\bmod p),
$$

and the first term in 4.15 is $\bar{c}_{1}^{a^{2}}$. In the generic situation $n \geq 2$ we argue similarly as in Case 1 that $\partial\left(\left(\Theta_{2}(z)\right)_{n+1} \cdots\left(\Theta_{2}(z)\right)_{p}\right)<\mu$ so that $\partial\left(\Theta_{2}\left(\Theta_{2}(z)\right)\right)<m$. It remains to deal with the special situation $n=1$, which makes use of the fact that the defining vectors satisfy $e_{i, p-1} \equiv 0$ for every $i \in\{1, \ldots, r\}$. For $m \geq 6$ the argument follows as before. For $m=4$, proceeding similarly, we obtain $\Theta_{2}(z)=\bar{c}_{1}^{a^{2}} \bar{c}_{2}^{a} \bar{c}_{3} \bar{c}_{4}^{a}$, so $\left(\Theta_{2}(z)\right)_{1}=b a^{w} c$ for some $b, c \in\left\langle b_{1}, \ldots, b_{r}\right\rangle$ and $w \in \mathbb{Z} / p \mathbb{Z}$. Thus subject to relabelling,

$$
\Theta_{1}\left(\Theta_{2}(z)\right)=\bar{c}_{1}^{a} \bar{c}_{2}^{a^{1-w}} \bar{c}_{3}^{a^{-w}} \bar{c}_{4} .
$$

As before, for $\partial\left(\Theta_{1}\left(\Theta_{2}(z)\right)_{1}\right)=2$, we need $\left(\bar{c}_{3}^{a^{*}}\right)_{1}$ to be a non-trivial element of $\left\langle b_{1}, \ldots, b_{r}\right\rangle$ and $\left(\bar{c}_{2}^{a^{*}}\right)_{1}$ to be a non-trivial power of $a$. Looking at the third term of (4.16), we require $w=-1$. However, then $\bar{c}_{2}^{a^{2}}$ contributes a trivial factor 1 to $\Theta_{1}\left(\Theta_{2}(z)\right)_{1}$ instead of a non-trivial power of $a$. Hence we see that the length decreases, as required.

\section{MAXIMAL SUBgroups}

In the present section we prove Theorem 1.2 about maximal subgroups of torsion multi-edge spinal groups. As in [12, it is convenient to phrase part of the argument in terms of proper dense subgroups with respect to the profinite topology.

5.1. Dense subgroups. We recall that the cosets of finite-index subgroups of a group $G$ form a base for the profinite topology on $G$. The group $G$ contains maximal subgroups of infinite index if and only if it contains proper dense subgroups with respect to the profinite topology. Indeed, a subgroup $H$ of $G$ is dense with respect to the profinite topology if and only if $G=N H$ for every finite-index normal subgroup $N$ of $G$. Therefore every maximal subgroup of infinite index in $G$ is dense and every proper dense subgroup is contained in a maximal subgroup of infinite index.

For the rest of the section, we fix a just infinite multi-edge spinal group $G=G_{\mathbf{E}}=\left\langle a, b_{1}, \ldots, b_{r}\right\rangle$ acting on the regular $p$-adic rooted tree $T$, for an odd prime $p$. Here $\mathbf{E}$ is the $r$-tuple of defining vectors $\mathbf{e}_{i}=\left(e_{i, 1}, \ldots, e_{i, p-1}\right)$, for $i \in\{1, \ldots, r\}$. For a vertex $u$ of $T$, we write

$$
G_{u}=U_{u}^{G} \cong G
$$


to denote the upper companion group at the vertex $u$. Every subgroup $M$ of $G$ gives rise to a subgroup

$$
M_{u}=U_{u}^{M} \leq G_{u} \cong G .
$$

Proposition 5.1 ([12, Proposition 3.2]). Let $T$ be a spherically homogeneous rooted tree and let $G \leq \operatorname{Aut}(T)$ be a just infinite group acting transitively on each layer of $T$. Let $M$ be a dense subgroup of $G$ with respect to the profinite topology. Then

(1) the subgroup $M$ acts transitively on each layer of the tree $T$,

(2) for every vertex $u \in T$, the subgroup $M_{u}$ is dense in $G_{u}$ with respect to the profinite topology.

The next result extends [12, Lemma 3.3], which addresses just infinite GGS-groups. Here we give a different and shorter proof for just infinite multi-edge spinal groups.

Proposition 5.2. Let $G$ be a just infinite multi-edge spinal group. Let $M$ be a proper dense subgroup of $G$, with respect to the profinite topology. Then $M_{u}$ is a proper subgroup of $G_{u}$ for every vertex $u$ of $T$.

Proof. Assume on the contrary, that there exists a vertex $u$ of $T$ such that $M_{u}=G_{u}$. Let $u$ be a vertex of minimal length $n$ with the specified property, and suppose $u=w x$ where $|w|=n-1$. By Proposition 5.1 and induction, $M_{w}$ is a proper dense subgroup of $G_{w}$. Since $G_{w}$ is isomorphic to $G$, we have $|u|=1$, say $u=u_{1}$ among the vertices $u_{1}, \ldots, u_{p}$ at level 1 .

Let $R=\operatorname{Rstab}_{M}(u)_{u}$. By our assumption, we have $R \unlhd M_{u}=G_{u}$. Since $G_{u} \cong G$ is just infinite, either $R$ has finite index in $G_{u}$, or $R$ is trivial.

Suppose first that $R$ has finite index in $G_{u}$. Then

$$
\begin{aligned}
|G: M| & \leq\left|G: \operatorname{Rstab}_{M}(1)\right|=\left|G: \operatorname{Stab}_{G}(1)\right|\left|\operatorname{Stab}_{G}(1): \operatorname{Rstab}_{M}(1)\right| \\
& \leq\left|G: \operatorname{Stab}_{G}(1)\right|\left|\prod_{i=1}^{p} G_{u_{i}}: \prod_{i=1}^{p} \operatorname{Rstab}_{M}\left(u_{i}\right)_{u_{i}}\right| \\
& \leq\left|G: \operatorname{Stab}_{G}(1)\right|\left|G_{u}: R\right|^{p}
\end{aligned}
$$

is finite. But, being a proper dense subgroup, $M$ has infinite index in $G$.

Hence $R$ is trivial, and so $\operatorname{Rstab}_{M}(1)$ is trivial. Thus

$$
\left|G / \operatorname{Rstab}_{G}(1)\right| \geq\left|M / \operatorname{Rstab}_{M}(1)\right|=|M|
$$

is infinite.

By Proposition 3.10, the group $G$ is not $\operatorname{Aut}(T)$-conjugate to the GGSgroup $\mathcal{G}$ in (3.7). Hence Proposition 3.7 shows that $G$ is a branch group. Thus $\operatorname{Rstab}_{G}(1)$ has finite index in $G$, a contradiction.

Proposition 5.3. Let $G=\left\langle a, b_{1}, \ldots, b_{r}\right\rangle$ be a just infinite multi-edge spinal group and $M$ a dense torsion subgroup of $G$, with respect to the profinite topology. For each $i \in\{1, \ldots, r\}$, there is a vertex $u$ of $T$ and an element $g \in \operatorname{Stab}_{G}(u)$ acting on $T_{u}$ as a for some $k \in \mathbb{Z} / p \mathbb{Z}$ under the natural identification of $T_{u}$ and $T$, such that 
(i) $\left(M^{g}\right)_{u}=\left(M_{u}\right)^{a^{k}}$ is a dense torsion subgroup of $G_{u} \cong G$,

(ii) there exists $b \in\left\langle b_{1}, \ldots, b_{r}\right\rangle$ with $\varepsilon_{b_{i}}(b) \neq 0$ such that $b \in\left(M_{u}\right)^{a^{k}}$.

Proof. Clearly, it suffices to prove the claim for $i=1$. Assuming that there are $u$ and $k$ such that (ii) holds, then as $G_{u} \cong G$ there exists $g \in \operatorname{Stab}_{G}(u)$ satisfying (i). Hence it suffices to show that such $u$ and $k$ exist.

Since $\left|G: G^{\prime}\right|$ is finite, $G^{\prime}$ is open in the profinite topology. Thus we find $x \in M \cap b_{1} G^{\prime}$. In particular, $x \in \operatorname{Stab}_{G}(1)$ with $\varepsilon_{b_{1}}(x) \not \equiv 0(\bmod p)$. We argue by induction on $\partial(x) \geq 1$.

First suppose that $\partial(x)=1$. Then $x$ has the form $x=b^{a^{k}}$, where $b \in\left\langle b_{1}, \ldots, b_{r}\right\rangle$ with $\varepsilon_{b_{1}}(b) \not \equiv 0(\bmod p)$ and $k \in\{0,1, \ldots, p-1\}$. Thus choosing the vertex $u$ to be the root of the tree $T$, we have $b \in\left(M_{u}\right)^{a^{-k}}$.

Now suppose that $\partial(x) \geq 2$. Recall from (4.4) and (4.11) the definition of $\varepsilon_{b_{1}}(x)$, and from (4.12) the definition of the maps $\varphi_{j}: \operatorname{Stab}_{G}(1) \rightarrow G_{u_{j}}$, where $u_{1}, \ldots, u_{p}$ denote the first level vertices of $T$. For any vertex $u$ of $T$, the subtree $T_{u}$ has a natural identification with $T$ and $G_{u} \cong G$. We freely use the symbols $a, b_{1}, \ldots, b_{r}$ to denote also automorphisms of $T_{u}$ under this identification. We claim that

$$
\varepsilon_{b_{1}}\left(\varphi_{1}(x)\right)+\ldots+\varepsilon_{b_{1}}\left(\varphi_{p}(x)\right) \equiv \varepsilon_{b_{1}}(x) \not \equiv 0 \quad(\bmod p) .
$$

To see this, write $x$ as a product of conjugates $b_{i}^{a^{*}}$ of the directed generators $b_{i}, i \in\{1, \ldots, r\}$, by powers $a^{*}$, where the symbol $*$ represents unspecified exponents. Then $\varepsilon_{b_{1}}(x)$ is the number of factors of the form $b_{1}^{a^{*}}$. Each of these factors contributes a directed automorphism $b_{1}$ in a unique coordinate, and none of the other factors $b_{2}^{a^{*}}, \ldots, b_{r}^{a^{*}}$ contributes a $b_{1}$ in any of the coordinates. Hence (5.1) holds.

By (5.1), there exists $j \in\{1, \ldots, p\}$ such that $\varepsilon_{b_{1}}\left(\varphi_{j}(x)\right) \not \equiv 0(\bmod p)$. Moreover, Lemma 4.4 shows that

$$
\partial\left(\varphi_{j}(x)\right) \leq\lceil\partial(x) / 2\rceil \leq(\partial(x)+1) / 2<\partial(x) .
$$

Suppose that $\tilde{x}=\varphi_{j}(x) \in M_{u_{j}}$ belongs to $\operatorname{Stab}_{G_{u(j)}}(1)$, where we write $u(j)=u_{j}$ for readability. By Proposition 5.1, the subgroup $M_{u_{j}}$ is dense in $G_{u_{j}} \cong G$. Since $\varepsilon_{b_{1}}(\tilde{x}) \not \equiv 0(\bmod p)$ and $\partial(\tilde{x})<\partial(x)$, the result follows by induction.

Now suppose that $\varphi_{j}(x) \notin \operatorname{Stab}_{G_{u(j)}}(1)$. For $\ell \in\{1, \ldots, p\}$ we claim that

$$
\varepsilon_{b_{1}}\left(\varphi_{\ell}\left(\varphi_{j}(x)^{p}\right)\right) \equiv \varepsilon_{b_{1}}\left(\varphi_{j}(x)\right) \not \equiv 0 \quad(\bmod p) .
$$

To see this, observe that $\varphi_{j}(x)$ is of the form

$$
\varphi_{j}(x)=a^{k} h,
$$

for $k \not \equiv 0(\bmod p)$ and $h \in \operatorname{Stab}_{G_{u(j)}}(1)$ with $\psi_{1}(h)=\left(h_{1}, \ldots, h_{p}\right)$, say. Hence raising $\varphi_{j}(x)$ to the prime power $p$, we get

$$
\varphi_{j}(x)^{p}=\left(a^{k} h\right)^{p}=h^{a^{(p-1) k}} h^{a^{(p-2) k}} \cdots h^{a^{k}} h,
$$


and thus for $\ell \in\{1, \ldots, p\}$,

$$
\varphi_{\ell}\left(\varphi_{j}(x)^{p}\right) \equiv h_{1} h_{2} \cdots h_{p} \quad\left(\bmod G_{u_{j \ell}}^{\prime}\right) .
$$

Here $u_{j \ell}$ denotes the $\ell$ th descendant of $u_{j}$. Arguing similarly as for (5.1), this yields

$$
\varepsilon_{b_{1}}\left(\varphi_{\ell}\left(\varphi_{j}(x)^{p}\right)\right) \equiv \varepsilon_{b_{1}}\left(h_{1}\right)+\ldots+\varepsilon_{b_{1}}\left(h_{p}\right) \equiv \varepsilon_{b_{1}}(h) \equiv \varepsilon_{b_{1}}\left(\varphi_{j}(x)\right) \quad(\bmod p)
$$

and $(5.3)$ holds.

Furthermore, we claim that

$$
\partial\left(\varphi_{\ell}\left(\varphi_{j}(x)^{p}\right)\right) \leq \partial\left(\varphi_{j}(x)\right)<\partial(x) .
$$

The second inequality comes from (5.2). To see that the first inequality holds, we note that

$$
\varphi_{\ell}\left(\varphi_{j}(x)^{p}\right)=\varphi_{\ell}\left(h^{a^{(p-1) k}}\right) \cdots \varphi_{\ell}\left(h^{a^{k}}\right) \varphi_{\ell}(h),
$$

and $\partial\left(\varphi_{j}(x)\right)=\partial(h)$. We write $h$ as a product of $\partial(h)$ conjugates $c_{j}^{a^{*}}$ of directed automorphisms $c_{j} \in\left\langle b_{1}, \ldots, b_{r}\right\rangle$, where the symbol $*$ represents unspecified exponents. Each factor $c_{j}^{a^{*}}$ contributes a directed automorphism $c_{j}$ in a unique coordinate and powers of $a$ in all other coordinates. Focusing on the $\ell$ th coordinate, we can write $\varphi_{\ell}\left(\varphi_{j}(x)^{p}\right)$ as a product of powers of $a$ and the $\partial(h)$ directed automorphisms $c_{j} \in\left\langle b_{1}, \ldots, b_{r}\right\rangle$. Hence (5.4) holds.

If $\tilde{x}=\varphi_{\ell}\left(\varphi_{j}(x)^{p}\right) \in M_{u_{j \ell}}$ belongs to $\operatorname{Stab}_{G_{u(j \ell)}}(1)$, we argue as follows. By Proposition 5.1, the subgroup $M_{u_{j \ell}}$ is dense in $G_{u_{j \ell}} \cong G$. Since $\varepsilon_{b_{1}}(\tilde{x}) \not \equiv 0$ $(\bmod p)$ and $\partial(\tilde{x})<\partial(x)$, the result follows by induction.

In general, we apply the operation $y \mapsto \varphi_{\ell}\left(y^{p}\right)$ more than once. Since $M$ is a torsion group, $x \in \operatorname{Stab}_{M}(1)$ and $\varphi_{j}(x)$ have finite order. Clearly, if $y \in G$ has finite order then $\varphi_{\ell}\left(y^{p}\right)$ has order strictly smaller than $y$. Thus after finitely many iterations, we inevitably reach an element

$$
\tilde{x}=\varphi_{\ell}\left(\varphi_{\ell}\left(\cdots \varphi_{\ell}\left(\varphi_{\ell}\left(\varphi_{j}(x)^{p}\right)^{p}\right)^{p} \cdots\right)^{p}\right) \in M_{u_{j \ell \cdots \ell}}
$$

which in addition to the inherited properties $\varepsilon_{b_{1}}(\tilde{x}) \not \equiv 0(\bmod p)$ and $\partial(\tilde{x})<$ $\partial(x)$ satisfies $\tilde{x} \in \operatorname{Stab}_{G_{u(j \ell \cdots \ell)}}(1)$. As before, the proof concludes by induction.

Recall the definition of the family $\mathcal{E}$ of groups by means of $(4.13)$.

Proposition 5.4. Let $G=\left\langle a, b_{1}, \ldots, b_{r}\right\rangle$ be a multi-edge spinal group. Suppose $G$ is not $\operatorname{Aut}(T)$-conjugate to a group in $\mathcal{E}$. Let $M$ be a dense subgroup of $G$, with respect to the profinite topology, and suppose that $b_{1} \in M$. Then there exist a vertex $u$ of $T$ and an element $g \in \operatorname{Stab}_{G}(u)$ acting on $T_{u}$ as $d \in\left\langle b_{1}, \ldots, b_{r}\right\rangle$ under the natural identification of $T_{u}$ and $T$, such that

(i) $\left(M^{g}\right)_{u}=\left(M_{u}\right)^{d}$ is a dense subgroup of $G_{u} \cong G$,

(ii) $a, b_{1} \in\left(M_{u}\right)^{d}$.

Proof. Akin to the proof of Proposition 5.3, it suffices to establish the existence of $u$ and $d$ such that (ii) holds. Observe that $G^{\prime}$ is open in $G$. Since $M$ is dense in $G$, there is $z \in G^{\prime}$ such that $a z \in M$. Write $\psi_{1}(z)=\left(z_{1}, \ldots, z_{p}\right)$. 
Let $u_{p}$ denote the $p$ th vertex at level 1 . The coordinate map $\varphi_{p}$ allows us to restrict $\operatorname{Stab}_{M}(1)$ to $M_{u_{p}}$. Clearly, $b_{1} \in M$ implies $b_{1}=\varphi_{p}\left(b_{1}\right) \in M_{u_{p}}$. Based on Lemma 3.3, we assume that the defining vector $\mathbf{e}_{1}$ for $b_{1}$ has first coordinate $e_{1,1}=1$. Consider the theta maps $\Theta_{1}, \Theta_{2}$ defined in Section 4.2. with reference to $b_{1}$. By their definition, $a \Theta_{1}(z)$ and $a \Theta_{2}(z)$ belong to $M_{u_{p}}$. Moreover, repeated application of $\varphi_{p}$ corresponds to repeated applications of $\Theta_{1}$ and $\Theta_{2}$. By Proposition 5.1 and Theorem 4.5, we may assume that $\partial(z) \in\{0,2\}$. If $\partial(z)=0$ we are done (with $d=1$ ).

Thus we may assume that $\partial(z)=2$ and we write $z=b^{-a^{m}} c^{a^{k}}$ for $b, c \in$ $\left\langle b_{1}, \ldots, b_{r}\right\rangle \backslash\{1\}$ and $m, k \in \mathbb{Z} / p \mathbb{Z}$ with $m \neq k$.

Case 1: $m, k \neq 1$. Here $z_{1}=a^{w}$ for some $w \in \mathbb{Z} / p \mathbb{Z}$. Thus $\Theta_{1}(z)=$ $\left[a, z_{1}^{-1}\right]=\left[a, a^{-w}\right]=1$, and $a \in M_{u_{p}}$.

Case 2: $m=1, k \neq 1$. Here

$$
\psi_{1}\left(b^{-a}\right)=\left(b^{-1}, *, \ldots, *\right) \quad \text { and } \quad \psi_{1}\left(c^{a^{k}}\right)=\left(a^{w}, *, \ldots, *\right),
$$

where $w \in \mathbb{Z} / p \mathbb{Z}$ and the symbols $*$ denote unspecified entries. Hence $z_{1}=b^{-1} a^{w}$ so that $\Theta_{1}(z)=\left[a, z_{1}^{-1}\right]=[a, b]$. This gives $a \Theta_{1}(z)=b^{-1} a b$. Remembering that $b_{1}$ and $b$ commute, we obtain $a, b_{1} \in\left(M_{u_{p}}\right)^{b^{-1}}$.

Case 3: $m \neq 1, k=1$. Here

$$
\psi_{1}\left(b^{-a^{m}}\right)=\left(a^{w}, *, \ldots, *\right) \quad \text { and } \quad \psi_{1}\left(c^{a}\right)=(c, *, \ldots, *),
$$

where $w \in \mathbb{Z} / p \mathbb{Z}$ and the symbols $*$ denote unspecified entries. Hence $z_{1}=$ $a^{w} c$ so that $\Theta_{1}(z)=\left[a, z_{1}^{-1}\right]=c^{a^{1-w}} c^{-a^{-w}}$. If $w \not \equiv-1(\bmod p)$, we are back in Case 1 or Case 2.

Suppose that $w \equiv-1(\bmod p)$. Then $\Theta_{1}(z)=c^{a^{2}} c^{-a}$, where

$$
c^{a^{2}}=(*, c, *, \ldots, *) \quad \text { and } \quad c^{-a}=\left(c^{-1}, *, \ldots, *\right)
$$

and the symbols $*$ denote unspecified powers of $a$. We recall from the definition of $\Theta_{2}$ that in the generic case $n \geq 2$ this gives $\Theta_{2}\left(\Theta_{1}(z)\right)=1$, hence $a, b_{1} \in M_{u_{p p}}$, where $u_{p p}$ is the $p^{2}$ th vertex at level 2 . In the special case $n=1$ we have $\Theta_{1}\left(\Theta_{1}(z)\right)=[a, c]$. In this case we proceed similarly as in Case 2.

Proposition 5.5. Let $G$ be a just infinite multi-edge spinal group. Suppose $G$ is not Aut $(T)$-conjugate to a group in $\mathcal{E}$. Let $M$ be a dense torsion subgroup of $G$, with respect to the profinite topology. Then there exists a vertex $u$ of $T$ such that $M_{u}=G_{u} \cong G$.

Proof. We first remark that for a vertex $u$ of $T$ and $g \in G$,

$$
\left(M^{g}\right)_{u^{g}}=G_{u^{g}} \Longleftrightarrow M_{u}=G_{u} .
$$

Now by Proposition 5.3, there exists a vertex $u_{1}$ of $T$ and an element $g \in \operatorname{Stab}_{G}\left(u_{1}\right)$ such that $x_{1} \in\left(M^{g}\right)_{u_{1}}$ with $x_{1} \in\left\langle b_{1}, \ldots, b_{r}\right\rangle$ and $\varepsilon_{b_{1}}\left(x_{1}\right) \neq 0$. We modify our generating set of directed automorphisms, by taking $\tilde{b}_{1}=x_{1}$ instead of $b_{1}$. Using (5.5) we may assume, without loss of generality, that $\tilde{b}_{1} \in M_{u_{1}}$. 
By Proposition 5.4, there exists a vertex $u_{1} v$ of $T_{u_{1}}$ and an element $h \in$ $\operatorname{Stab}_{G}\left(u_{1} v\right)$ with $a, b_{1} \in\left(M^{h}\right)_{u_{1} v}$. Once more by (5.5), we may assume that $a, \tilde{b}_{1} \in M_{u_{1} v}$.

Applying Proposition 5.3 again, we see that there exists $k \in \mathbb{Z} / p \mathbb{Z}$ and a vertex $u_{1} v u_{2}$ of $T_{u_{1} v}$ such that $x_{2} \in\left(M_{u_{1} v u_{2}}\right)^{a^{k}}$ with $x_{2} \in\left\langle\tilde{b}_{1}, b_{2}, \ldots, b_{r}\right\rangle$ and $\varepsilon_{b_{2}}\left(x_{2}\right) \neq 0$. Note that $\varepsilon_{b_{2}}$ is now defined with respect to the new generating set $\tilde{b}_{1}, b_{2}, \ldots, b_{r}$ of directed automorphisms. Since $a, \tilde{b}_{1} \in M_{u_{1} v}$, it follows that $a, \tilde{b}_{1} \in M_{u_{1} v u_{2}}$; recall Proposition 3.1 with respect to the multi-edge spinal group $\left\langle a, \tilde{b}_{1}\right\rangle$. In particular, $\left(M_{u_{1} v u_{2}}\right)^{a^{k}}=M_{u_{1} v u_{2}}$. Hence, replacing $b_{2}$ by $\tilde{b}_{2}=x_{2}$, we obtain $a, \tilde{b}_{1}, \tilde{b}_{2} \in M_{u_{1} v u_{2}}$. Continuing in this manner, we arrive at a vertex $u=u_{1} v u_{2} \cdots u_{r}$ such that $a, \tilde{b}_{1}, \ldots, \tilde{b}_{r} \in M_{u}$, where $a, \tilde{b}_{1}, \ldots, \tilde{b}_{r}$ is a generating set for $G_{u}$.

Theorem 5.6. Let $G=\left\langle a, b_{1}, \ldots, b_{r}\right\rangle$ be a just infinite multi-edge spinal group. Suppose $G$ is not Aut $(T)$-conjugate to a group in $\mathcal{E}$. Then $G$ does not contain any proper dense torsion subgroups, with respect to the profinite topology.

Proof. Suppose on the contrary that $M$ is a proper dense torsion subgroup of $G$, with respect to the profinite topology. By Proposition 5.2, for every vertex $u \in T$ we have $M_{u}$ is properly contained in $G_{u}$. However, by Proposition 5.5, the subgroup $M_{u}$ is all of $G \cong G_{u}$. This gives the required contradiction.

Since $\mathcal{E}$ does not contain any torsion groups and every torsion multi-edge spinal group is a $p$-group, Theorem 1.2 is a direct consequence of Theorem 5.6.

We finish by proving Corollary 1.3 .

Proof of Corollary 1.3. Suppose that $G=G_{\mathbf{E}}=\left\langle a, b_{1}, \ldots, b_{r}\right\rangle$, the multiedge spinal group associated to some defining vectors $\mathbf{E}$. By Theorem 1.2, all maximal subgroups of $G$ have finite index. Since $G$ is residually finite and just infinite, its chief factors are finite. Hence [7, Lemma 3] shows that, if $K$ is a subdirect subgroup of some direct product $G \times \ldots \times G$ of copies of $G$, then all maximal subgroups of $K$ are of finite index.

Let $H$ be a group commensurable with $G$, and fix a finite index subgroup $L$ of $H$ that is isomorphic to a finite index subgroup $K$ of $G$. For $i \in\{1, \ldots, r\}$, we write $G_{i}=\left\langle a, b_{i}\right\rangle$ for the GGS-subgroup of $G$, generated by $a$ and $b_{i}$. By [10], each of these GGS-groups $G_{i}$ has the congruence subgroup property. Since $K \cap G_{i}$ has finite index in $G_{i}$, there is $m_{i} \in \mathbb{N}$ such that

$$
\operatorname{Stab}_{G_{i}}\left(m_{i}\right) \subseteq K \cap G_{i} \subseteq K .
$$

Since $\operatorname{Stab}_{G_{i}}\left(m_{i}\right)$ is subdirect in $G_{i} \times p^{m_{i}} . \times G_{i}$, we conclude that, for $m=$ $\max \left\{m_{i} \mid 1 \leq i \leq r\right\}$, the group $\operatorname{Stab}_{K}(m)$ is subdirect in $G \times ?^{m} . \times G$.

As observed above, this implies that all maximal subgroups of $\operatorname{Stab}_{K}(m)$ are of finite index. Since $\operatorname{Stab}_{K}(m)$ has finite index in $K$ and $L \cong K$ has 
finite index in $H$, we deduce from [7, Lemma 1] that all maximal subgroups of $H$ have finite index.

Acknowledgement. Special cases of the results in this paper form part of Alexoudas' $\mathrm{PhD}$ thesis [1].

\section{REFERENCES}

[1] T. Alexoudas, Maximal subgroups of branch groups, $\mathrm{PhD}$ thesis, Royal Holloway, University of London, 2013.

[2] L. Bartholdi, R. I. Grigorchuk and Z. Šunik, Handbook of algebra 3, North-Holland, Amsterdam, 2003.

[3] I. V. Bondarenko, Finite generation of iterated wreath products, Arch. Math. (Basel) 95(4) (2010), 301-308.

[4] G. A. Fernández-Alcober and A. Zugadi-Reizabal, GGS-groups: Order of congruence quotients and Hausdorff dimension, Trans. Amer. Math. Soc. 366 (2014), 1993-2017.

[5] R. I. Grigorchuk, On Burnside's problem on periodic groups, Funktsional. Anal. $i$ Prilozhen 14 (1) (1980), 53-54.

[6] R. I. Grigorchuk, Just infinite branch groups, in: New horizons in pro-p groups, Birkhäuser, Boston, 2000.

[7] R. I. Grigorchuk and J. S. Wilson, A structural property concerning abstract commensurability of subgroups, J. London Math. Soc. 68(2) (2003), 671-682.

[8] N. Gupta and S. Sidki, On the Burnside problem for periodic groups, Math. Z. 182 (3) (1983), 385-388.

[9] D. S. Passman, The semiprimitivity of group algebras, in Methods in ring theory (Levico Terme, 1997), Lecture Notes in Pure and appl. Math. 198, Dekker, New York, 1998.

[10] E. L. Pervova, Profinite completions of some groups acting on trees, J. Algebra 310 (2) (2007), 858-879.

[11] E. L. Pervova, Everywhere dense subgroups of a group of tree automorphisms, $\operatorname{Tr}$. Mat. Inst. Steklova 231 (Din. Sist., Avtom. i. Beskon. Gruppy) (2000), 356-367.

[12] E. L. Pervova, Maximal subgroups of some non locally finite p-groups, Internat. J. Algebra Comput. 15(5-6) (2005), 1129-1150.

[13] A. V. Rozhkov, On the theory of groups of Aleshin type, Mat. Zametki 40 (5) (1986), 572-589.

[14] S. Sidki, A primitive ring associated to a Burnside 3-group, J. London Math. Soc. 55 (2) (1997), 55-64.

[15] T. Vovkivsky, Infinite torsion groups arising as generalizations of the second Grigorchuk group, in: Algebra (Moscow, 1998), de Gruyter, Berlin, 2000, 357-377.

Theofanis Alexoudas: Department of Mathematics, Royal Holloway, UniVERsity of London, Egham TW20 0EX, UK

E-mail address: Theofanis.Alexoudas.2009@live.rhul.ac.uk

Benjamin Klopsch: Mathematisches Institut, Heinrich-Heine-Universität, 40225 Düsseldorf, Germany

E-mail address: klopsch@math.uni-duesseldorf.de

Anitha Thillaisundaram: Mathematisches Institut, Heinrich-Heine-Universität, 40225 DÜSSELDORF, GERMANY

E-mail address: anitha.t@cantab.net 